\title{
Expert consensus on neoadjuvant immunotherapy for non-small cell lung cancer
}

\author{
Wenhua Liang ${ }^{1}$, Kaican Cai ${ }^{2}$, Chun Chen ${ }^{3}$, Haiquan Chen ${ }^{4}$, Qixun Chen ${ }^{5}$, Junke Fu ${ }^{6}$, Jian $\mathrm{Hu}^{7}$, \\ Tao Jiang ${ }^{8}$, Wenjie Jiao ${ }^{9}$, Shuben $\mathrm{Li}^{1}$, Changhong Liu ${ }^{10}$, Deruo Liu ${ }^{11}$, Wei Liu ${ }^{12}$, Yang Liu ${ }^{13}$, Haitao $\mathrm{Ma}^{14}$, \\ Xiaojie Pan ${ }^{15}$, Guibin Qiao ${ }^{16}$, Hui Tian ${ }^{17}$, Li Wei ${ }^{18}$, Yi Zhang ${ }^{19}$, Song Zhao ${ }^{20}$, Xiaojing Zhao ${ }^{21}$, \\ Chengzhi Zhou ${ }^{1}$, Yuming Zhu ${ }^{22}$, Ran Zhong ${ }^{1}$, Feng Li ${ }^{1}$, Rafael Rosell ${ }^{23}$, Mariano Provencio ${ }^{24}$, \\ Erminia Massarelli ${ }^{25}$, Mara B. Antonoff ${ }^{26}$, Toyoaki Hida ${ }^{27}$, Marc de Perrot ${ }^{28}$, Steven H. Lin ${ }^{29}$, \\ Massimo Di Maio ${ }^{30}$, Antonio Rossi ${ }^{31}$, Dirk De Ruysscher ${ }^{32}$, Robert A. Ramirez ${ }^{33}$, \\ Wolfram C. M. Dempke ${ }^{34}$, D. Ross Camidge ${ }^{35}$, Nicolas Guibert ${ }^{36}$, Raffaele Califano ${ }^{37}$, Qi Wang ${ }^{38}$, \\ Shengxiang Ren $^{39}$, Caicun Zhou ${ }^{39}$, Jianxing $\mathrm{He}^{1}$
}

${ }^{1}$ Department of Thoracic Surgery and Oncology, The First Affiliated Hospital of Guangzhou Medical University, National Center for Respiratory Medicine, State Key Laboratory of Respiratory Disease and National Clinical Research Center for Respiratory Disease, Guangzhou, China; ${ }^{2}$ Nanfang Hospital, Guangzhou, China; ${ }^{3}$ Fujian Medical University Union Hospital, Fuzhou, China; ${ }^{4}$ Fundan University Shanghai Cancer Center, Shanghai, China; ${ }^{5}$ Zhejiang Cancer Hospital, Hangzhou, China; ${ }^{6}$ The First Affiliated Hospital of Xi'an Jiaotong University, Xi'an, China; ${ }^{7}$ The First Affiliated Hospital, Zhejiang University, School of Medicine, Hangzhou, China; ${ }^{8}$ Tangdu Hospital, Xi'an, China; ${ }^{9}$ The Affiliated Hospital of Qingdao University, Qingdao, China; ${ }^{10}$ The Second Hospital of Dalian Medical University, Dalian, China; ${ }^{11}$ China-Japan Friendship Hospital, Beijing, China; ${ }^{12}$ The First Bethune Hospital of Jilin University, Changchun, China; ${ }^{13}$ Chinese PLA General Hospital, Beijing, China; ${ }^{14}$ The Affiliated Hospital of Soochow University, Suzhou, China; ${ }^{15}$ Fujian Provincial Hospital, Fuzhou, China; ${ }^{16}$ Guangdong Provincial People's Hospital, Guangzhou, China; ${ }^{17}$ Qilu Hospital of Shandong University, Ji'nan, China; ${ }^{18}$ Henan Provincial People's Hospital, Zhengzhou, China; ${ }^{19}$ Xuanwu Hospital Capital Medical University, Beijing, China; ${ }^{20}$ The Affiliated Hospital of Zhengzhou University, Zhengzhou, China; ${ }^{21}$ Renji Hospital, Shanghai Jiao Tong University School of Medicine, Shanghai, China; ${ }^{22}$ Shanghai Pulmonary Hospital, Shanghai, China; ${ }^{23}$ Department of Medical Oncology, University of Barcelona, Hospital de Badalona Germans Trias i Pujol, Barcelona, Spain; ${ }^{24}$ Hospital Universitario Puerta de Hierro-Majadahonda, Madrid, Spain; ${ }^{25}$ City of Hope National Cancer Center, Duarte, CA, USA; ${ }^{26}$ Department of Thoracic and Cardiovascular Surgery, University of Texas MD Anderson Cancer Center, Houston, TX, USA; ${ }^{27}$ Department of Thoracic Oncology, Aichi Cancer Center Hospital, Nagoya, Japan; ${ }^{28}$ Toronto Mesothelioma Research Program, Toronto General Hospital, Toronto, ON, Canada; ${ }^{29}$ Department of Radiation Oncology, The University of Texas M.D. Anderson Cancer Center, Houston, TX, USA; ${ }^{30}$ Department of Oncology, University of Turin/Division of Medical Oncology, Ordine Mauriziano Hospital, Turin, Italy; ${ }^{31}$ Oncology Center of Excellence, Therapeutic Science \& Strategy Unit, IQVIA, Milan, Italy; ${ }^{32}$ Department of Radiation Oncology (MAASTRO), GROW-School for Oncology and Developmental Biology, Maastricht University Medical Centre, Maastricht, The Netherlands; ${ }^{33}$ Department of Internal Medicine, Section of Hematology/Oncology, Ochsner Medical Center, Kenner, LA, USA; ${ }^{34}$ Department of Hematology and Oncology, University Medical School, Munich, Germany; ${ }^{35}$ Division of Medical Oncology, University of Colorado Anschutz Medical Campus, Aurora, CO, USA; ${ }^{36}$ Thoracic Oncology Department, Larrey Hospital, University Hospital of Toulouse, Toulouse, France; ${ }^{37}$ Department of Medical Oncology, The Christie NHS Foundation Trust, and Division of Cancer Sciences, The University of Manchester, Manchester, UK; ${ }^{38}$ Department of Respiratory Medicine, The Second Hospital, Dalian Medical University, Dalian, China; ${ }^{39}$ Department of Medical Oncology, Shanghai Pulmonary Hospital, Tongji University School of Medicine, Shanghai, China

Correspondence to: Jianxing He. National Center for Respiratory Medicine, China State Key Laboratory of Respiratory Disease and National Clinical Research Center for Respiratory Disease, The First Affiliated Hospital of Guangzhou Medical University, Guangzhou, China. Email: drjianxing.he@gmail.com.

Submitted Oct 21, 2020. Accepted for publication Dec 24, 2020.

doi: $10.21037 /$ tlcr-2020-63

View this article at: http://dx.doi.org/10.21037/tlcr-2020-63 


\section{Introduction}

Lung cancer is the leading cause of cancer-related death worldwide and in China (1). According to the statistics of the National Cancer Center of China, there were 733,300 new cases of non-small cell lung cancer (NSCLC) and approximately 610,200 related deaths in 2015 (2). For patients with early staged disease, surgery is the mainstay of treatment, and it is commonly followed by adjuvant chemotherapy for patients with locally advanced resectable NSCLC. Although complete surgical resection may be curative for NSCLC, 25-70\% of patients (with different proportion according to stage) eventually relapse despite complete resection (3). Platinum-based adjuvant chemotherapy has been shown to marginally increase the 5-year survival rate of patients by 4-8\% (4-6). Even after treatment with surgery and indicated adjuvant therapies in eligible cases, approximately $20-30 \%$ of stage I, $50 \%$ of stage II, and $60 \%$ of stage IIIA patients still die within 5 years (7). In the past decade, experts have conducted a number of investigations on the perioperative management of resectable NSCLC; however, progress remains slow, and patients still have a high risk of recurrence and death.

Neoadjuvant therapy is defined as any therapy delivered prior to definitive local therapy intended to increase the cure rate. It provides several theoretical benefits in managing such patients with NSCLC. In the setting of, neoadjuvant therapy given prior to radical surgery this approach can also have the goals of downstaging, improving the resection rate, and more promptly treating subclinical micro-metastases than adjuvant approaches, delivered after the definitive local therapy. In addition, the compliance with neoadjuvant therapy has been shown to be better than in the adjuvant setting, and the biological effect of the neoadjuvant therapy can be analyzed directly in the resected tumor specimens (8). A meta-analysis on patients with stage IB-IIIA NSCLC that compared chemotherapy plus subsequent surgery $v s$. surgery alone showed that the 5-year survival rate was $5 \%$ higher after receiving neoadjuvant chemotherapy (NCT) (9). Therefore, the comprehensive NSCLC data suggest that, for resectable NSCLC, NCT improves survival compared with surgery alone but appear to show no significant survival benefit compared with adjuvant chemotherapy (10).

In the last 5 years, immune checkpoint inhibitors (ICIs) have profoundly changed the treatment paradigm for patients with advanced NSCLC (11-15). Immunotherapy has provided hope for long-term survival benefits to a minority of patients with metastatic lung cancer. For treatment-naive patients with driver mutationnegative NSCLC, the 5 -year survival rate of single agent pembrolizumab was $23.2 \%$; for the previously treated patients with driver mutation-negative NSCLC, the 5 -year survival rates of single agent pembrolizumab and nivolumab were $15.5 \%$ and $16 \%$, respectively $(16,17)$. Given the profound impact made by immunotherapy drugs for patients with advanced disease, significant attention has been directed in recent years toward investigating the potential role for early-stage NSCLC patients, and whether they, too, can achieve long-term benefits from the inclusion of immunotherapy into their treatment algorithms.

Many phase Ib/II clinical trials have reported promising results, and a series of large-scale phase III clinical trials are underway. However, these various investigations have employed different strategies of neoadjuvant immunotherapy, in terms of the specific regimens as well as number of treatment cycles (18). To better guide Chinese thoracic surgeons in the neoadjuvant immunotherapy of NSCLC, well-known thoracic surgeons in China participated in an in-depth discussion on the hot topics and controversial issues of neoadjuvant immunotherapy and formed the Expert consensus on neoadjuvant immunotherapy for non-small-cell lung cancer by incorporating the latest evidence on neoadjuvant immunotherapy.

\section{Consensus 1: preoperative use of neoadjuvant immunotherapy with or without platinum-based chemotherapy for patients with resectable stage IB-IIIA NSCLC may be considered}

In 1994, Rosell and Roth published two classic prospective randomized controlled trials of NCT for stage IIIA NSCLC, and since then a number of studies since have demonstrated that, for early-stage NSCLC, the efficacy of preoperative NCT plus surgery is similar to that of surgery plus postoperative adjuvant chemotherapy, and both strategies including systemic treatment are better than surgery alone in terms of overall survival (OS) (9,19-21). Due to the relevant toxicity of neoadjuvant/adjuvant chemotherapy, patient tolerance can, at times, be poor. Compared with surgery alone, the addition of neoadjuvant or adjuvant chemotherapy only modestly improves the 5 -year OS rate by about $5 \%(9,21)$. Thus, there is a clear need for well-tolerated and effective neoadjuvant and adjuvant therapies for resectable NSCLC. Compared with conventional chemotherapy, immunotherapy is better 
tolerated in most patients, and treatment-related toxicities may have minimal influence on ability to complete surgical resection. Immunotherapy is being actively explored for the perioperative management of NSCLC.

When programmed death 1 (PD-1)/programmed deathligand 1 (PD-L1) inhibitors kill tumors, antigen-presenting cells (APC) are needed so that the tumor cells can be recognized by host $\mathrm{T}$ cells. By blocking the PD-1/PDL1 interaction with inhibitory antibodies, immunotherapy allows the activated $\mathrm{T}$ cells to release cytokines, perforines, granzymes and others to kill tumor cells. In terms of mechanisms involved, when the tumor volume is relatively large, APC bears a relatively large antigen load, which elicits a stronger antitumor $\mathrm{T}$ cell response. Therefore, neoadjuvant immunotherapy could theoretically be better than adjuvant immunotherapy and may yield an even greater survival long-term benefit (22). Preclinical studies support these hypotheses. In animal models, compared with adjuvant immunotherapy, neoadjuvant immunotherapy resulted in greater prolongation of median survival time and a higher survival rate (23-25).

No data from large-scale phase III clinical trials of neoadjuvant immunotherapy are yet available, but the results of several phase II clinical trials showed that neoadjuvant immunotherapy may play an important role in the multi-disciplinary management of early-stage NSCLC (26-31). The results of trials of neoadjuvant immunotherapy were summarized in Table 1.

CheckMate-159 is a phase II clinical trial which evaluated the safety and feasibility of a preoperative neoadjuvant immunotherapy with two-cycle nivolumab monotherapy, in resectable stage I-IIIA NSCLC. This single-arm study enrolled 22 patients, of which 20 patients received two cycles of the experimental treatment and 20 underwent complete surgical resection. This study showed that neoadjuvant nivolumab monotherapy was safe, tolerable and effective. The major pathologic response (MPR, defined as residual viable tumor cells less than 10\%) rate was $45 \%$ (26). The phase II clinical trial, TOP1501, was designed to evaluate the safety and feasibility of another preoperative neoadjuvant immunotherapy, two cycles of pembrolizumab monotherapy, in patients with stage IB-IIIA NSCLC. This monotherapy was also found to be safe and efficacious. The MPR rate was $28 \%$, and up to $80 \%$ of patients had pathological remission $\geq 50 \%$ (28). The LCMC3 phase II clinical study of PDL1 inhibitors showed that in patients with stage IB-IIIA NSCLC (plus selected stage IIIB patients), two-cycle atezolizumab monotherapy was found to be well-tolerated and have promising outcomes. The MPR rate was $19 \%$, the pathologic complete remission (pCR, defined as no residual viable tumor) was $5 \%$, and the MPR was unrelated to PD-L1 expression (27). In addition to the above immunomonotherapies, neoadjuvant immunotherapy combined with chemotherapy is associated with higher MPRs in cross-trial comparisons and a suggestion of longer OS utilizing the same cross-trial comparators. A phase II study by Shu et al. (32) aimed to investigate the safety and efficacy of preoperative neoadjuvant immunotherapy in the form of four cycles of atezolizumab monotherapy plus carboplatin plus albumin-bound paclitaxel in patients with stage IB-IIIA NSCLC, and the results revealed that this treatment algorithm was feasible, tolerable, and beneficial. The MPR was $50 \%$, and the pCR reached $21.4 \%$. The NADIM study presented at ASCO in 2019 aimed to assess the safety and efficacy of preoperative neoadjuvant immunotherapy in the form of three-cycle nivolumab monotherapy plus carboplatin plus paclitaxel in patients with stage IIIA (N2 or T4N0) NSCLC, and results were promising. The MPR was $83 \%$, the pCR was $71 \%$, and tumor was down-staged in $90 \%$ of the patients (33). Currently, the experimental groups of all phase III clinical trials are including neoadjuvant immunotherapy with PD-1/PD-L1 inhibitors plus platinum-based doublet chemotherapy, such as CheckMate-816 (NCT02998528), KEYNOTE-671 (NCT03425643), IMpower-030 (NCT03456063), AEGEAN (NCT03800134), CheckMate-77T (NCT04025879). The ongoing trials were summarized in Table S1.

In summary, this consensus suggests that preoperative use of neoadjuvant immunotherapy with or without platinumbased chemotherapy might be considered for patients with resectable stage IB-IIIA NSCLC.

\section{Consensus 2: there is no evidence that molecular markers uniformly predict the efficacy of neoadjuvant immunotherapy so that biomarker-based selection is not essential. However, it should be cautious to use neoadjuvant single agent immunotherapy in patients with potentially negative factors, such as epidermal growth factor receptor (EGFR)- sensitive mutation/ALK fusion}

PD-1/PD-L1 inhibitors have changed the treatment strategies of advanced NSCLC, but we know that not all 


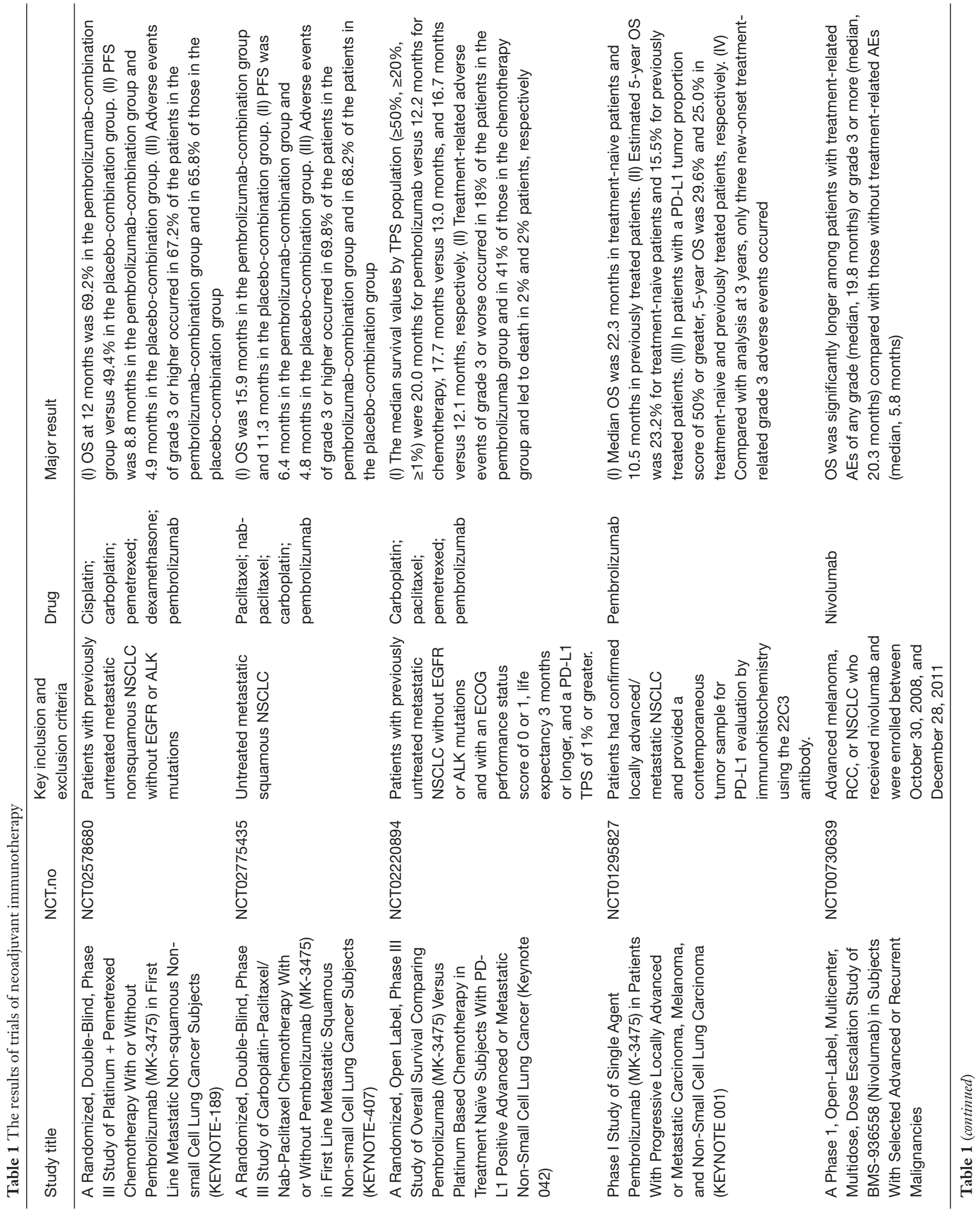



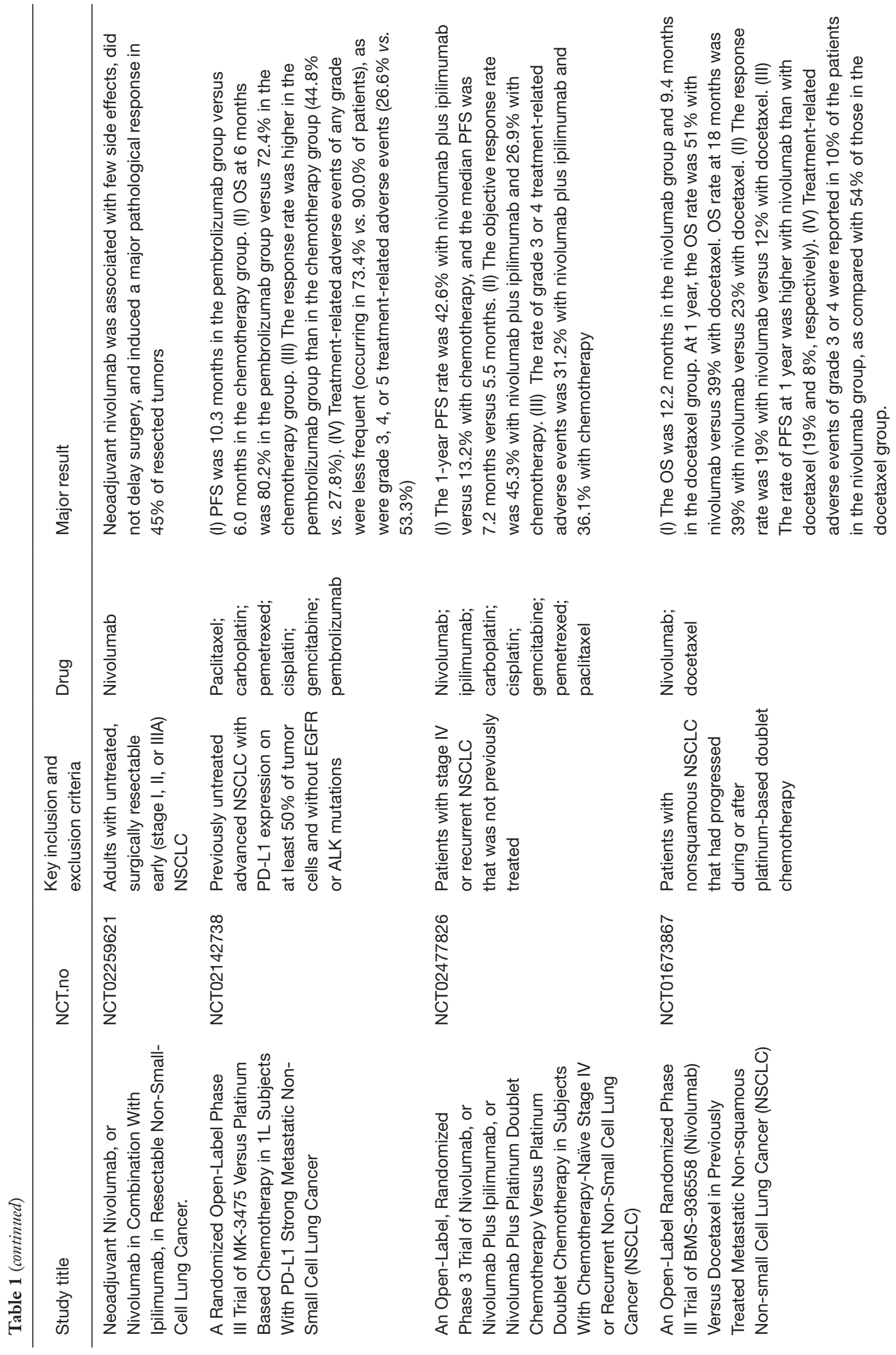

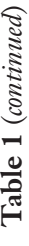




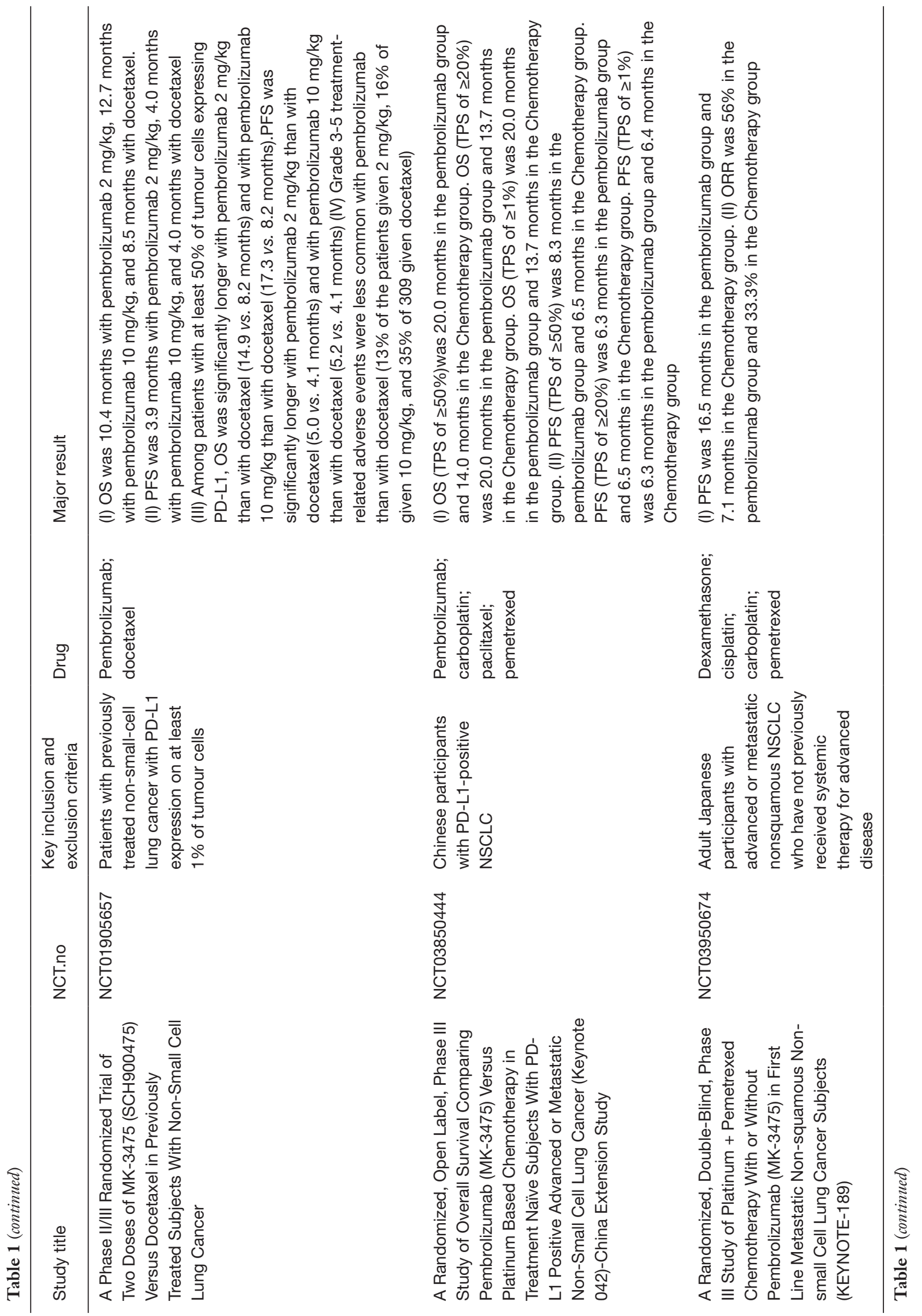




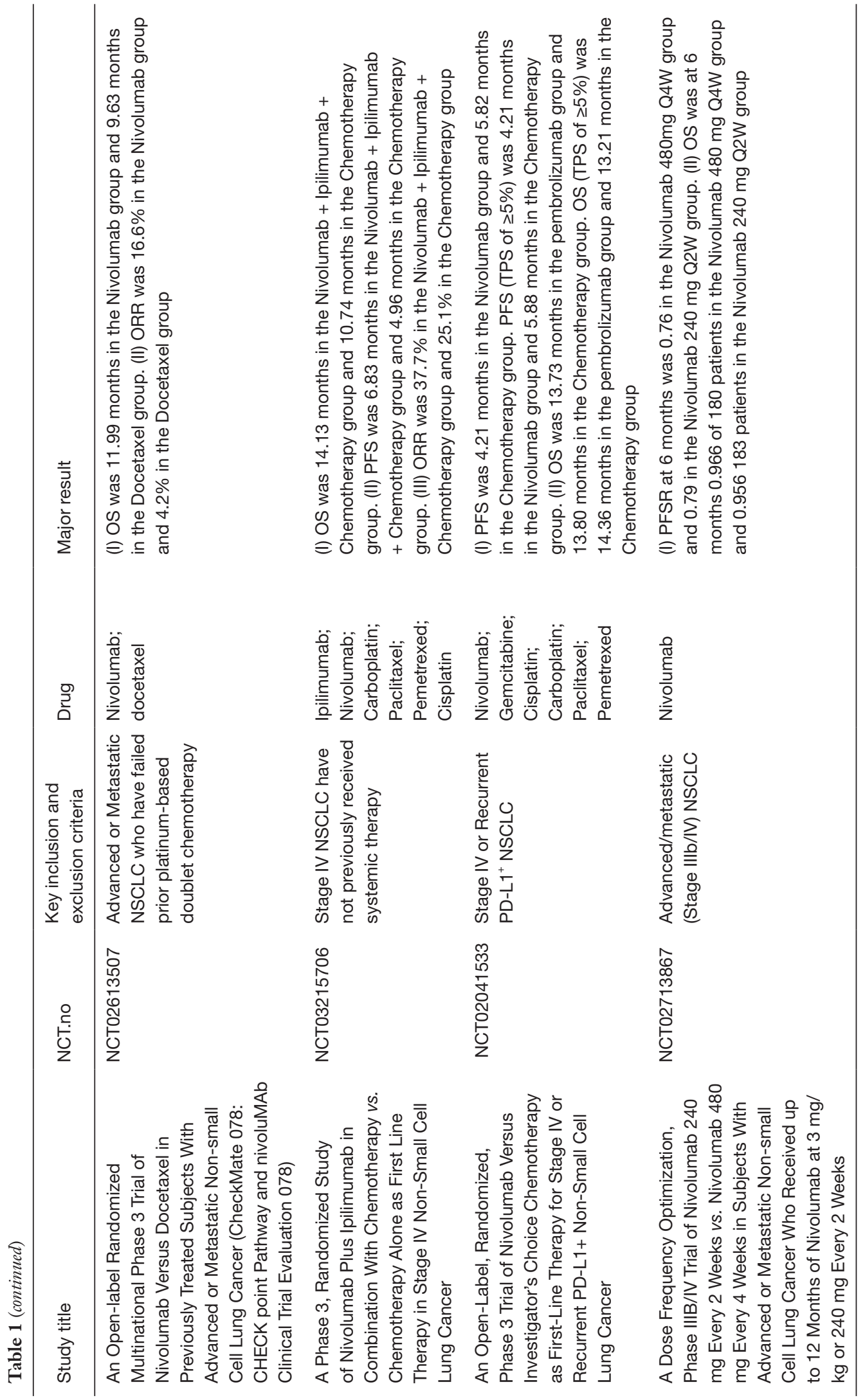


patients can benefit from them. For patients with driver mutation-negative NSCLC, the response rate of PD-1/ PD-L1 inhibitor monotherapy has been approximately $14-20 \%$, and that of first-line monotherapy in advanced NSCLC patients with PD-L1 expression $\geq 50 \%$ is approximately $50 \%$ (34). Therefore, it is critical to identify predictive biomarkers to select patients who can benefit from PD-1/PD-L1 inhibitors, avoiding the treatment to those patients who will not benefit. The most promising marker as such is PD-L1 so far. Supported by wellfounded evidence. KEYNOTE-024 and KEYNOTE-042 studies indicated that among patients with high or positive PD-L1 expression, pembrolizumab monotherapy was superior to the standard chemotherapy. The higher the PD-L1 expression, the more benefit the patients received from single agent immunotherapy. Therefore, all guidelines consistently recommend PD-L1 as the companion diagnostic for patients with driver mutationnegative NSCLC receiving immunotherapy $(13,35)$. The IMpower110 study also showed that among the patients with driver mutation-negative NSCLC and high PD-L1 expression in tumor cells and/or interstitial cells (TC3/IC3), atezolizumab monotherapy was superior to the standard chemotherapy (36). Some results of KEYNOTE-016, KEYNOTE-164, KEYNOTE-1022, KEYNOTE-028, KEYNOTE-177 and KEYNOTE-158 indicated that the objective remission rate of pembrolizumab monotherapy reached $39.6 \%$ in solid-tumor patients with microsatellite instability high (MSI-H) or mismatch repair deficiency. Based on this finding, the United States Food and Drug Administration (FDA) approved pembrolizumab monotherapy for patients with disease progression after previous treatment or metastatic solid tumor patients with MSI-H or mismatch repair deficiency, which is the first indication for cancer treatment based on biomarkers (37). However, these features are very rare in NSCLC.

The use of PD-L1 as a biomarker, however, remains to be complicated by a number of factors including the variability in tissue collection timing, the antibody and methodology used for staining (including the definition of positivity and the non-standardised test design), the heterogeneity and dynamic of PD-L1 expression within different tumors, and the role of PD-L1 expression on tumor-infiltrating lymphocytes and other immune cells versus the malignant cell population. In addition, PD-L1 is regarded to be a biological continuum and therefore might be of limited value as a biomarker in this subset of patients. Pembrolizumab (anti-PD-1) is approved (EMA, FDA) for first-line treatment of NSCLC patients with advanced or metastatic cancers (with $\mathrm{PD}-\mathrm{L} 1$ expression $\geq 50 \%$ using the Dako 22C3 IHC assay), whereas for second-line treatment a PD-L1 expression of $\geq 1 \%$ is required. Both, nivolumab (anti-PD-1) and atezolizumab (anti-PD-L1) are also approved (EMA, FDA) in the first- and second-line setting, but PD-L1 screening is only mandatory for atezolizumab, however, complementary PD-L1 diagnostics are approved for NSCLC. Durvalumab (anti-PD-L1) is currently for stage non-resectable IIIA/IIIB NSCLC, PD-L1 testing is recommended. To date, many groups of oncologists are attempting to establish better predictive biomarkers in NSCLC for monoclonal antibodies targeting the PD-1/ PD-L1 axis to select patients who might have a greater benefit from immune checkpoint therapies.

In addition to PD-L1 and MSI-H, tumor mutation burden (TMB) is also considered a potential biomarker for immunotherapy. Noteworthy, TMB can be assessed in different ways and, as a continuous variable, 'high' can also be variably defined. Although the retrospective analyses of KEYNOTE-042 and KEYNOTE-189 trials, and the prospective study CheckMate-227 all showed that TMB could not effectively predict the efficacy of immunotherapy alone or immunotherapy combined with chemotherapy as first-line treatment of advanced NSCLC (38), the KEYNOTE-158 results showed that, among patients with disease progression or metastases after previous treatment, the outcomes of single-agent pembrolizumab in those with high TMB were significantly better than in those with low TMB. Based on this, the FDA approved the pembrolizumab monotherapy for patients with high TMB as defined by $\geq 10$ mutations $/ \mathrm{Mb}$ and disease progression after previous treatment and for metastatic solid tumor patients (39).

The IMpower 150 trial is the first phase III study to demonstrate a clinically meaningful and significant PFS benefit with atezolizumab plus bevacizumab and chemotherapy (paclitaxel and carboplatin) versus bevacizumab plus chemotherapy in the first-line stetting of advanced or metastatic NSCLC (8.3 versus 6.8 months, HR $=0.62, \mathrm{P}<0.0001)$ and the PFS benefit was seen regardless of the PD-L1 status in all patients. The PFS benefit, however, was even more pronounced in patients expressing a $\mathrm{T}$ effector gene signature (11.3 versus 6.8 months, $H R=0.51$, $\mathrm{P}<0.0001)$ indicating that the expression of gene signatures may be more robust to predict clinical response following treatment with PD-L1 inhibitors (40).

Of note, in experimental systems with NSCLC-bearing xenografts, the tumor microenvironment (TME) has also 
been found to significantly contribute to the response to CPIs. Several lines of evidence have suggested that tumor-associated macrophages (TAMs: positivity for PDL1, CD133, and CD163) within the TME can reduced responses to immunotherapies by suppressing CD8positive cytotoxic $\mathrm{T}$ cells (CTLs) They are also regarded to be responsible for the hyperprogression following immunotherapy (41). In addition, cancer-associated fibroblasts (CAFs) have also been shown to suppress responses to CPIs by decreasing CD8-positive CTLs. Ford et al. (42) have provided the first evidence that TGF- $\beta$ can stimulate NADPH-Oxidase 4 (NOX-4) which in turns produces reactive oxygen species that can activate CAFs and thereby confer resistance to IO treatments. Although so far these are experimental approaches, it is likely that the CAF and TAM status will guide immunotherapy treatment strategies in the neoadjuvant setting in the future as well.

Differently from advanced NSCLC, immunotherapy for early-stage NSCLC is still under investigation. Clinical studies have yet to find biomarkers that can predict the efficacy of neoadjuvant immunotherapy $(27,43)$. The NEOSTAR study showed that, when using neoadjuvant nivolumab monotherapy or nivolumab combined with ipilimumab monotherapy to treat stage I-IIIA NSCLC, the baseline PD-L1 expression in patients obtaining complete/ partial response $(\mathrm{CR} / \mathrm{PR})$ was higher than that in patients obtaining stable/progressive disease (SD/PD), and the baseline PD-L1 expression in patients obtaining MPR was higher than those who did not (43). In the LCMC3 study, neoadjuvant atezolizumab monotherapy was given to patients with stage IB-IIIB NSCLC. Pathological remission and MPR were observed regardless of the expression level of PD-L1, and TMB had no correlation with pathological remission and MPR (27). Immunotherapy for early-stage NSCLC is still under investigation, and biomarkers in this setting are also being explored. All currently completed studies are small-scale phase I/II clinical trials, and the role of biomarkers, including PD-L1, MSI-H, $\mathrm{T}_{\text {eff }}$ cell signatures, and $\mathrm{TMB}$, remains unclear, warranting further exploration.

Over the past 20 years, with the discovery of driver genes such as EGFR, ROS, RET, and $A L K$ and the development of targeted agents, the treatment of NSCLC has entered the era of precision drug therapy, greatly improving the prognosis of molecularly selected subgroups of patients with advanced NSCLC (44). As described above, ICIs can bring long-term benefits to patients with driver mutationnegative NSCLC (11-13). Therefore, many researchers have explored whether ICIs can also bring long-term benefits to NSCLC patients with driver mutations.

CheckMate-057 is a phase III clinical study aiming to explore the efficacy of nivolumab monotherapy as the second-line treatment of advanced nonsquamous NSCLC, and its efficacy was compared to that of docetaxel. In this study, 82 EGFR-mutated NSCLC patients and 21 patients with $A L K$ translocation were included, and they all had previously received the first-line platinum-based doublet chemotherapy. Subgroup analysis of EGFR-mutated NSCLC patients showed that OS and progression-free survival (PFS) were not improved in patients receiving nivolumab monotherapy compared to those receiving docetaxel (45). Another III randomized clinical trial, KEYNOTE-010, comparing pembrolizumab vs. docetaxel, performed subgroup analysis on EGFR-mutated NSCLC patients and showed that pembrolizumab did not improve OS compared to docetaxel (46). A recent, real-world, retrospective analysis showed that EGFR-mutated and $A L K$-rearranged NSCLC patients did not benefit from PD-1/PD-L1 monotherapy. A number of clinical trials and retrospective studies have shown that for advanced EGFRmutated or $A L K$-rearranged patients, the chance of benefit with immunotherapy is modest, and the addition of ICIs on the corresponding targeted therapies does not bring an additional benefit but increases the occurrence of toxic and side effects. Further support for this proposal came from the observation that EGFR mutations may decrease PD-L1 expression (47).

In addition to the poor efficacy of immunotherapy in NSCLC patients with driver mutations (positive EGFR and $A L K$ ), genes such as STK11 that are tumor suppressor genes are frequently mutated in NSCLC and may reduce cluster of differentiation (CD) $8^{+} \mathrm{T}$ cell density or function, thus affecting the tumor-related immune response through multiple pathways (48). The MYSTIC study showed that the prognosis of patients with STK11mutated NSCLC receiving single-agent durvalumab was poor (49). The exploratory analysis of KEYNOTE-042 showed that regardless of the STK11 mutation status, the patients who received pembrolizumab monotherapy showed better PFS and OS than the patients who received standard chemotherapy (50). However, complete responses to nivolumab in patients with STK11 mutation have been also reported (51). At present, it is not clear whether STK11 mutation is a prognostic factor or a predictive factor in patients with NSCLC receiving for PD-1/PD-L1 inhibitor therapy. 
Although the roles of driver genes, such as $E G F R$ and $A L K$, and inhibitory genes, such as STK11, in immunotherapy for early-stage NSCLC are still not clear. Based on the above data, patients with potentially unfavorable factors such as EGFR-sensitive mutation or ALK fusion should be cautious to receive neoadjuvant single agent immunotherapy.

\section{Consensus 3: for neoadjuvant immunotherapy, two to four cycles are recommended, and after every two cycles, review and evaluation should be performed to update the treatment plan}

The purpose of neoadjuvant immunotherapy is to enhance downstaging, improve the $\mathrm{R} 0$ resection rate, and subsequently treat subclinical micrometastases. A short course of neoadjuvant immunotherapy may not be adequate for immunotherapy to have an effect, but if the duration of immunotherapy is too long, tumor progression may lead to the loss of surgical window of opportunity, so the length of neoadjuvant immunotherapy is very important (52). A preclinical study showed that the presence of a primary tumor appeared to be key to the efficacy of neoadjuvant immunotherapy, and the efficacy was closely correlated with the timing of tumor resection after neoadjuvant immunotherapy (53). To prevent progression in patients with drug resistance, the International Neoadjuvant Melanoma Consortium (INMC) recommends six to eight weeks of neoadjuvant therapy for melanoma, depending on the cycle length of the clinical trial (54). At present, the effects of neoadjuvant immunotherapy on early-stage NSCLC are known only from phase I/II clinical trials, without large-scale phase III trials. The neoadjuvant single agent immunotherapy in CheckMate159, LCMC3, and TOP1501, was performed for two cycles, and surgery was performed 28-56 days after the first cycle (26-28). Neoadjuvant immunotherapy combined with chemotherapy (NADIM: phase II with $\mathrm{N}=51$ ) or combination of two checkpoint inhibitors (PD-1 and CTLA-4) (NEOSTAR: phase II with $\mathrm{N}=44$ ) was performed for three to four cycles, and surgery was performed 3-7 weeks after the end of the neoadjuvant therapy (43). Currently, the role of neoadjuvant immunotherapy in early-stage NSCLC is still not fully elucidated, and review after every two cycles is recommended to assess tumor remission and update the treatment plan. However, as there is a low predictive value of CT scans or of RECIST criteria, in case no distant metastases are found, patients should proceed to surgical resection if still feasible.

In summary, two to four cycles are recommended for neoadjuvant immunotherapy, and re-evaluate after every two cycles is recommended to refine the treatment plan.

\section{Consensus 4: the benefit from neoadjuvant immunotherapy should be preferably assessed by positron-emission tomography (PET)- computed tomography (CT), in conjunction with serum tumor markers and/or circulating tumor DNA (ctDNA) load}

Tumor shrinkage after the treatment is clear evidence for the antitumor activity of neoadjuvant therapy, and the objective remission rate is an important indicator to evaluate tumor shrinkage and antitumor activity. CT is often used to assess the response of NSCLC patients to neoadjuvant therapy. The Response Evaluation Criteria in Solid Tumors (RECIST) by CT is an important predictor of OS in NSCLC patients after NCT (55). However, the histopathological response of $41-45 \%$ patients may be inconsistent with the CT evaluation $(26,56)$. Changes in inflammation and interstitial or fibrotic components of tumors may affect the CT results, leading to the inability of CT imaging to accurately predict histopathological responses after neoadjuvant therapy. When using imaging examinations, the efficacy is determined by continuously measuring changes in tumor size in these patients, which has inherent limitations. Therefore, conventional imaging combined with metabolic imaging may be required to determine efficacy (56). Some investigators have suggested that PET-CT may be more advantageous in the evaluation of neoadjuvant therapy because the uptake of ${ }^{18} \mathrm{~F}$-fluorodeoxyglucose by the tumor is closely related to the proliferative activity and the number of remaining vital tumor cells. Multiple PET-CT studies have shown that PET-CT can effectively evaluate the efficacy of neoadjuvant therapy. Although the response to neoadjuvant therapy as seen on PET-CT may be related to an improved prognosis, the role of PET-CT in neoadjuvant therapy at early stage still requires further exploration $(31,55,57)$. In addition, incorporating serum tumor markers or ctDNA may increase the accuracy of tumor load assessment $(58,59)$. This is particularly important for assessment of neoadjuvant immunotherapy, given that discrepancy between radiologic and pathologic response, as well as pseudo progression, have been extensively reported $(24,28)$. 
In summary, the benefit of neoadjuvant immunotherapy is preferred to be assessed by PET-CT, in conjunction with tumor markers and/or ctDNA load. However, more research on the accuracy and the need for standardization, especially for ctDNA measurements, of these modalities is needed before they can be introduced in standard of care.

\section{Consensus 5: surgery can be performed 4-6 weeks after the last cycle of neoadjuvant immunotherapy}

It is very difficult to determine the timing of surgery after neoadjuvant immunotherapy. For neoadjuvant chemotherapy, early surgery may lead to serious surgical complications, while delayed surgery may lead to tumor progression (52). However, neoadjuvant immunotherapy is distinct from neoadjuvant chemotherapy in terms of adverse events. Before determining the optimal time for neoadjuvant immunotherapy and surgery, it is important to understand the $\mathrm{T}$ cell amplification cycle, determine the best time for effector cells to exert their effects, and time the tumor resection for when the impact on antitumor immunity will be the least. Experimentally, this may be very challenging, though recent studies have shown that it is possible to measure the human antigen-specific $T$ cell response over time through systematic deuterium labeling, but further basic and clinical trials are still needed to determine the optimal timing of surgery (60). Although the results of neoadjuvant immunotherapy on early-stage NSCLC are all from phase I/II clinical trials, they still have reference value. CheckMate 159, LCMC3, and TOP1501 included two cycles of neoadjuvant single agent immunotherapy, and surgery was performed 28 to 56 days after the first cycle, i.e., 1-5 weeks after the end of immunotherapy (26-28). Neoadjuvant immunotherapy combined with chemotherapy (NADIM, NCT02716038) or the combination of two immunotherapies (NEOSTAR) was performed for three to four cycles, and surgery was performed 3-7 weeks after the end of the neoadjuvant immunotherapy (43). In summary, surgery is recommended at 4-6 weeks after the last neoadjuvant immunotherapy cycle.

\section{Consensus 6: There is no definitive evidence that neoadjuvant immunotherapy affects the conduct or safety of surgery}

The morbidity and safety of surgery after neoadjuvant immunotherapy is still not definitively known. Some clinical trials have demonstrated that after neoadjuvant radiotherapy (NRT) or NCT, surgery is safe and feasible, but that neoadjuvant immunotherapy may lead to tissue adhesion, thus increasing the operative difficulty. Thus, appropriate concerns have been raised as to whether neoadjuvant immunotherapy may increase the surgery complexity $(61,62)$. Currently, the phase I/II studies of neoadjuvant immunotherapy showed that the incidence of any adverse events caused by neoadjuvant single agent immunotherapy was approximately $57 \%$, the incidence of adverse events $\geq$ grade 3 was approximately $4.5-8 \%$, and the completion rate of planned surgery was $78-100 \%$, which are all similar to those of NCT and NRT (26-28). The NEOSTAR study assessed surgical difficulty and pulmonary function after neoadjuvant immunotherapy, and the results showed that ICIs had less impact on the surgical resection rate and surgical complexity and had no adverse effect on perioperative outcomes (43). A retrospective analysis of 19 patients in the United States showed that, for metastatic or unresectable patients, pneumonectomy after neoadjuvant immunotherapy was feasible, with a high $\mathrm{R} 0$ rate, while surgery may be challenging, serious complications were rare (63). There are still no clear conclusions about the impact and safety of neoadjuvant immunotherapy on surgical procedures, and more data are needed.

In summary, based on the available data, there is no conclusive evidence that neoadjuvant immunotherapy adversely affects surgical procedures or their safety.

\section{Consensus 7: pathological remission (MPR, pCR) needs to be assessed, recorded, and reported by specialized pathologist after neoadjuvant immunotherapy}

In a prospective multicenter phase II study of stage IIIA and IIIB NSCLC in 1997, Junker et al. (64) developed a tumor regression grading system based on resection specimens (62 cases) from 28 patients with lung squamouscell carcinoma and 12 patients with lung adenocarcinoma after combined NCT and NRT. The results were compared to spontaneous regressive changes in a control group of 50 untreated NSCLC patients. The proposed three-level grading system of pathological response included grade I (no or only slight tumor regression), grade IIA (significant but incomplete tumor regression, more than $10 \%$ vital tumor 
tissue), grade IIB (less than $10 \%$ vital tumor tissue), and grade III (complete tumor regression without vital tumor tissue). Patients with grade IIB or III tumor regression had a much longer survival time than those with grade II or IIA tumor regression (64). Later, Junker et al. improved the grading criteria for tumor regression and defined grade I as no tumor regression or only spontaneous tumor regression, grade II as treatment-induced tumor regression, grade IIa as more than $10 \%$ vital tumor tissue, grade IIb as less than $10 \%$ vital tumor tissue, and grade III as complete tumor regression. The authors also found that along with the complete resection of the tumor, treatment-induced tumor regression and less than $10 \%$ vital tumor tissue were essential for improving long-term outcomes (65). However, many later trials questioned whether the percentage of vital tumor tissue remaining after NCT in NSCLC patients was associated with prognosis. The response of lung squamouscell carcinoma to NCT was significantly better than that of lung adenocarcinoma, the median percentages of vital tumor tissue were $40 \%$ and $60 \%$, respectively, and the critical MPR values were $26 \%$ and $12 \%$, respectively (66). In 2017, the College of American Pathologists still recommended MPR as the study endpoint of clinical trials on neoadjuvant immunotherapy for lung cancer. Currently, MPR is defined as neoadjuvant therapy-induced tumor regression with less than $10 \%$ vital tumor tissue, and pCR is defined as neoadjuvant therapy-induced complete tumor regression without vital tumor tissue (67).

Because ICIs have only been used in neoadjuvant therapy for NSCLC in recent years, most of the current results come from phase I/II clinical trials. The MPR of neoadjuvant single agent immunotherapy is in the range of $19-45 \%$, and that of immunotherapy combined with neoadjuvant therapy fluctuates within 33-83\% (26-28,43). These studies are small, the results have not been verified in phase III clinical trials, and the relationships of MPR with PFS and OS need further confirmation. However, immunotherapy has brought higher MPR and surgical resection rates. The current clinical trials of neoadjuvant immunotherapy can collect more specimens for further exploration.

In summary, pathological remission (MPR, pCR) needs to be assessed, recorded and reported by specialized pathologist after neoadjuvant immunotherapy.
Consensus 8: for neoadjuvant immunotherapy in nonprogressive patients, immunotherapy can be resumed after surgery, and it can be maintained for 1 year

PD-1/PD-L1 inhibitors have greatly altered the treatment strategies of advanced NSCLC. The Chinese Society of Clinical Oncology (CSCO) guidelines recommend that advanced NSCLC patients with positive PD-L1 expression and negative driver mutations can be treated with pembrolizumab monotherapy, and the immunotherapy can be withdrawn at disease progression or 35 cycles (2 years). Advanced NSCLC patients whose tumors do not harbor driver mutations, regardless of PD-L1 expression, can be treated with pembrolizumab monotherapy combined with chemotherapy for 4 cycles, followed by maintenance pembrolizumab monotherapy for a total of 2 years. The CSCO guidelines also recommend 1-year maintenance therapy of durvalumab after concurrent chemoradiotherapy for stage III unresectable NSCLC patients. Currently, there is no definitive recommendation on immune maintenance therapy after neoadjuvant immunotherapy, and it varies from no postoperative immune maintenance therapy to 1 -year immune maintenance therapy $(26-28,43)$.

Recently, Antonia et al. reported the results of the phase III PACIFIC study (NCT02125461) (68). In this study the role of immune checkpoint blockade with durvalumab in locally advanced, unresectable, stage III NSCLC was evaluated. Eligible patients had NSCLCs without progression after they had been treated with at least two cycles of platinum-based chemotherapy concurrent with radiotherapy (chemo-radiotherapy) at a dose of 54 to $66 \mathrm{~Gy}$. A total of 713 patients were randomly assigned in a 2:1 ratio to receive either durvalumab $(10 \mathrm{mg} / \mathrm{kg})$ or placebo every 2 weeks for up to 12 months. Results showed that the coprimary end point of median PFS was 16.8 months in the durvalumab group versus 5.6 months in the placebo group (HR $=0.52 ; 95 \% \mathrm{CI}, 0.42-0.65)$. In addition, the ORR (assessed by blinded independent central review) was found to be higher in the durvalumab group than in the placebo group $(28.4 \%$ vs. $16.0 \%, \mathrm{P}<0.001)$. Interestingly, clinical benefit was observed irrespectively of NSCLC tumor stage (IIIA or IIIB), histologic type, or geographic distribution. Most notably, however, brain metastases developed far more frequently in the placebo group as in the durvalumab group 
$(11.0 \%$ vs. $5.5 \%)(16,17)$. The OS was also significantly increased with not reached versus 29.1 months $(H R=0.68)$.

Given the experience with immunotherapy in advanced NSCLC and stage III unresectable NSCLC, the neoadjuvant immunotherapy of nonprogressive patients can be resumed after surgery, and can be maintained for 1 year.

\section{Consensus 9: immunotherapy or induction chemotherapy can be offered in borderline resectable locally advanced NSCLC, and consideration for surgery should be reevaluated upon restaging}

The standard treatment for stage IIIA/B unresectable NSCLC is maintenance therapy with durvalumab monotherapy after concurrent chemoradiotherapy (based on the results of the PACIFIC Trial). Debate exists as to whether these patients are best served by surgery after induction therapy. The ESPATUE study ( $\mathrm{N}=246$ of 500 planned, trial had been stopped due to slow recruitment) showed that some patients with stage III unresectable disease may benefit from induction chemotherapy or chemoradiotherapy: their $\mathrm{T}$ and $\mathrm{N}$ stages were significantly downgraded, and tumors became surgically resectable with a downgrade rate of $44 \%$. Patient characteristics were balanced between the two arms, in which 81 were assigned to surgery ( $\operatorname{arm~A}$ ), and 80 were assigned to a chemoradiotherapy boost. In arm B, $81 \%$ underwent R0 resection. With a median follow-up after random assignment of 78 months, 5 -year OS and PFS did not differ between arms. OS rates of $44 \%$ for arm B and $40 \%$ for arm A (log-rank $\mathrm{P}=0.34)$ and PFS rates of $32 \%$ for arm B and $35 \%$ for arm A $(\log$-rank $\mathrm{P}=0.75)$. OS at 5 years was $34.1 \%$ (95\% CI, 27.6-40.8\%) in all 246 patients, and 216 patients $(87.8 \%)$ received definitive local treatment (69).

Most recently Reck et al. (70)provided the first evidence that the combination of immunotherapy followed by chemotherapy and subsequently maintenance therapy resulted in a significantly improved mOS (CheckMate-9LA). In this phase III trial patients $(\mathrm{N}=719)$ were randomized between immunotherapy with ipilimumab and nivolumab (plus two cycles of platinum-based chemotherapy) followed by ipilimumab plus nivolumab as maintenance until progression versus four cycles of platinum-based chemotherapy followed by maintenance therapy (until progression). The ORR rate for the immunotherapy arm was $38 \%$ ( $2 \%$ complete remission), mOS rates after one year were $63 \%$ versus $47 \%$ (HR =0.66) (approved by FDA and EMA). The results were seen regardless of the PDL1 status. From this trial the authors concluded that the IO-chemotherapy combination should be considered $m$ effective and well-tolerated novel treatment opportunity for advanced and/or metastatic NSCLC. Although this trial was conducted in stage IV NSCLC patients, the ORR of $38 \%$ (including 2\% CR rate) is remarkable and further studies are currently planned to evaluate this regimen in earlier stages of NSCLC in the neoadjuvant setting.

Although surgical resection did not increase the postoperative PFS and OS compared to radical chemoradiotherapy, selected patients (T3N2, T4N0/T4N1) had a significant long-term survival benefit in the subgroup analysis, and the benefit in the cohort of patients with stage III B (T4N0-1) patients was the most significant (69). Although many immunotherapy neoadjuvant clinical trials are still underway, the NADIM study indicated that after stage III (N2 or T4N0/N1) patients received four cycles of nivolumab monotherapy combined with chemotherapy, mPFS was found to be $77.1 \%$ after 24 months. $30 \%$ of patients had treatment-related adverse events of grade 3 or worse; however, none of the adverse events were associated with surgery delays or deaths. The downstage rate was $90.2 \%$, the completion rate of planned surgery was $89.1 \%$, and the MPR rate was $83 \%$, indicating a promising longterm survival benefit in this group of patients (71).

In summary, induction immunotherapy with or without chemotherapy can be considered for unresectable locally advanced NSCLC (particularly in selected N2 and T4 patients), and after the tumor stage is downgraded, the possibility of surgery could be reevaluated. The results of randomized phase III trials with OS as the primary endpoint should be awaited before this strategy is introduced into standard of care.

\section{Key questions and perspectives}

\section{Do you use neoadjuvant PD-1/PD-L1 blockade in routine} practice? Why or why not?

Rafael Rosell: Neoadjuvant PD-L1 approach is still not standardized, therefore I prefer to include patients in available clinical trials on immune checkpoint inhibitors in combination with chemotherapy. However, understanding that there are patients who may not have access to clinical trials, the use of preoperative neoadjuvant immunotherapy could be adequate for stage IIIA NSCLC patients. 
Mariano Provencio: I believe that the results of chemoimmunotherapy could support its use in clinical practice, it makes all the same sense as in advanced stages and the results are much better than just chemotherapy. Perhaps we should wait for the ongoing trials, but their adoption should be quick and perhaps accept surrogates of pathological response... but it is not approved in my country.

Erminia Massarelli: Yes, I use it within clinical trials of neoadjuvant immunotherapy.

I truly believe that checkpoint inhibitors alone or plus chemotherapy can significantly downstage tumors and I believe the advantage especially in stage II-III NSCLC is significant. However, so far we are only using it within clinical trials.

Mara B. Antonoff: We try to enroll as many patients as possible onto trials evaluating neoadjuvant PD-L1 blockade; however, outside of a clinical trial, we do not routinely use it in the neoadjuvant setting as it is not yet approved for use outside of a trial.

Toyoaki Hida: We don't use neoadjuvant PD-1/PD-L1 blockade in routine practice, because immune checkpoint inhibitors (ICIs) are not yet approved for the use of preoperative treatment in lung cancer in Japan. We use ICIs for preoperative treatment in clinical trials only.

Marc de Perrot: I do not use neoadjuvant PD-L1 blockade in routine practice due to the lack of access. Neoadjuvant IO is part of clinical trials in our institution.

Steven H. Lin: No, all our patients with this approach are treated on clinical trial, since it is currently not a standard approach for the use of neoadjuvant immunotherapy although early studies have shown promising major pathologic response. All neoadjuvant approaches should be done on the ongoing numerous single arm or randomized trials. Neoadjuvant chemotherapy is still a good option as the control arm in those randomized trials.

Massimo Di Maio: We do not currently use neoadjuvant $\mathrm{PD}-1 / \mathrm{PD}-\mathrm{L} 1$ blockade in routine practice, because this is not a standard approach according to our national guidelines by AIOM (Italian Association of Medical Oncology). In general, neoadjuvant treatment is not commonly used for patients who are judged eligible for surgery, with the exception of a known positivity of mediastinal lymph nodes, when a neoadjuvant chemotherapy is commonly considered before re-evaluation with the surgeon.

As a general rule, Italy has a publicly funded health system, and only treatments approved and reimbursed can be considered in clinical practice. Furthermore, AIOM has chosen to produce recommendations only for drugs/ treatments that are reimbursed by the national health system, considering that it would make no sense to recommend something that cannot be used in clinical practice.

At the moment, use of neoadjuvant PD-1/PD-L1 blockade is limited to participation in clinical trials.

Antonio Rossi: I do not use neoadjuvant PD-1/PDL1 blockade in routine practice because, despite the very interesting results coming from phase I/II trials, it is still an experimental approach. The Site in which I worked had no possibility to be involved in trials addressing this issue in order to participate to the investigation of this strategic approach. Randomized trials are ongoing and if the results will be positive this approach should be evaluated by regulatory agencies and then considered for clinical practice.

Dirk de Ruysscher: No, only in the context of a clinical trial. There are no phase III data showing an increase of OS.

Robert A. Ramirez: No. The data looks promising for combination chemo/IO in the neoadjuvant setting, however, outside of a clinical trial we don't have large scale studies to guide us. Plus, in the US we run into reimbursement issues and cannot get this in the neoadjuvant setting.

Wolfram C. M. Dempke: In our institution neoadjuvant therapies are currently not used routinely as they are not FDA/EMA approved so far (with very few exceptions). However, we participate in clinical studies.

D. Ross Camidge: NO-It is not licensed and its OS benefit is not clear. It needs to be compared with adjuvant therapy as it will cause delay in surgery.

Nicolas Guibert: I do, but only within clinical trials because no ICIs as neoadjuvant therapies are approved in France yet.

Raffaele Califano: It's not in routine practice as not approved/funded.

\section{Do you think biomarker is necessary in choosing neoadjuvant PD-1/PD-L1 blockade therapy? If yes, which ones?}

Rafael Rosell: Needless to say, immune biomarkers are desirable and a PD-L1 tumor proportion score (TPS) of more than $50 \%$ could serve and encourage patients. However, patients with a TPS of lower than $50 \%$, but more than $1 \%$, are also suitable for the neoadjuvant immunotherapy approach. Furthermore, if liquid biopsy is accessible, a high blood TMB is also orientative to predict 
response to immune checkpoint inhibitors. NSCLC with driver mutations or gene fusions cannot be considered for neoadjuvant immunotherapy approaches.

Mariano Provencio: No, I don't think so.

Erminia Massarelli: No, I do not think current biomarkers are needed considering that PD-L1 negative patient have good benefit as well. I believe we still need to discover reliable markers of response in $\mathrm{PD}-\mathrm{L} 1<$ or $=50 \%$.

Mara B. Antonoff: We know that PD-L1 status can help determine patients who are likely to do well, and we also know that patients with EGFR and ALK mutations are not likely to derive benefit, and would not include such patients on these trials when they can receive targeted therapy instead.

Toyoaki Hida: Yes: We think biomarker may be necessary in choosing neoadjuvant PD-1/PD-L1 blockade therapy in the case of single ICI use.

Candidate for biomarker: PD-1 CD8 T cells, PD-1 regulatory $\mathrm{T}$ (Treg) cells, frequency of PD-1 CD8 T cells relative to that of PD-1 Treg cells.

It may be not necessary in the cases of ICI use in combination with platinum-doublet chemotherapy, although it would be better to have biomarkers that can predict the effect.

Marc de Perrot: PD-L1 expression on tumor cell is a good biomarker when PD-1/PD-L1 is used as single therapy. However, biomarker may not be necessary when PD-1/PDL1 blockade is combined with chemotherapy.

Steven H. Lin: We select for patients who shouldn't get immunotherapy, which are patients who have an oncogene driven mutation such as EGFR, ALK, ROS, RET. We don't select for other markers like PD-L1 status.

Massimo Di Maio: Very good question. It probably depends if we are talking about single agent PD-1/PD-L1 blockade or combination with chemotherapy. If combined with chemotherapy, based on the results obtained in the advanced setting, we could avoid a pre-treatment selection according to PD-L1 expression. In fact, we know that chemotherapy is an acceptable and effective strategy as neoadjuvant treatment, so the risk of undertreatment would be limited. On the other hand, in the case of single-agents, we know that a not negligible proportion of patients would be at risk of not obtaining a response, so a selection should be probably considered (with the only exception of a very short planned duration of treatment, which would not delay the timing of surgery), probably based on the PD-L1 expression. Unfortunately, evidence about biomarkers' role in this setting (including PD-L1 expression but also TMB) is not robust.

Antonio Rossi: Biomarkers should drive the treatment for any stage of NSCLC disease. Unfortunately, we do not have biomarkers for every subgroup of NSCLC patients. However, with the available data, in the neoadjuvant setting, no specific biomarker can help in defining the subgroup of patients which could much benefit by neoadjuvant immunotherapy. The potential role of PD-L1 expression and other potential biomarkers such as MSI or TMB, in this setting should be evaluated more extensively in the ongoing randomized trials, in order to have the possibility to select NSCLC patients who could much benefit from neoadjuvant immunotherapy.

Dirk de Ruysscher: At present, we do not have a good marker for concurrent chemo-PD-1/PD-L1 blockage, while for anti-PD-1/PD-L1 alone, tumor PD-L1 expression is still the best. There are many new developments such as the T-cell (CD8 and Treg) density on the initial tumor biopsy, which are promising.

Robert A. Ramirez: No. I suppose if someone were PDL1 $>50 \%$, MSI high or high TMB that may sway me to use single agent IO, however, we run into lack of data as above. We also know the RR in the combination chemo/IO is higher in the metastatic setting regardless of biomarkers.

Wolfram C. M. Dempke: This is a controversial discussion. We follow the label of the IOs (have added this in the manuscript as well), but truly believe that additional biomarkers are urgently warranted (for new examples see my paragraph within the manuscript).

D. Ross Camidge: I suspect it may influence outcomes the obvious cases would be ALK and EGFR.

Nicolas Guibert: No clear biomarker of response (including TMB and PD-L1) has been identified yet in the neoadjuvant setting like it has been well stated in the manuscript.

I would thus not recommend the use of biomarker to guide neoadjuvant ICI yet.

I would, however, like proposed in the manuscript, suggest to avoid neoadjuvant ICI monotherapy in patients with EGFR, ALK or STK11 alterations, given the low response rates observed in these populations in advanced stages.

This extrapolation may however need to be validated in clinical trials.

More endeavors are however needed to better select patients.

Raffaele Califano: PD-L1 using single agent PD-1/PDL1 blockade. No need for biomarker if using neoadjuvant 
Chemo + IO.

\section{How do/will you determine the cycles of neoadjuvant PD-1/PD-L1 blockade therapy?}

Rafael Rosell: At present, there is no pre-specified number of cycles. Two to four cycles are the recommendation.

Mariano Provencio: We use 3 cycles.

Erminia Massarelli: Usually Two to three cycles as per clinical trials guidelines.

Mara B. Antonoff: We believe anywhere from 2-4 cycles is beneficial, and the data don't yet define an optimal number. We don't use it outside of a clinical trial, so at this time, we use it for the designated amount of time based on the clinical trial. We need further data from these studies to decide the exact number of cycles.

Toyoaki Hida: 2-3 cycles depending on the degree of bone marrow suppression (degree of irAE).

Marc de Perrot: Two to four cycles are sufficient in the neoadjuvant setting.

Steven H. Lin: Standardly it has been as little as one cycle to 2 cycles, +/- chemotherapy. Neoadjuvant chemoradiation with IO is being explored as well.

Massimo Di Maio: I would determine the cycles of neoadjuvant PD-1/PD-L1 blockade according to the evidence produced by clinical trials.

In the case of combination with chemotherapy, I would have no problem to plan 3 or 4 cycles of treatment, considering that the risk of undertreatment should be negligible. In the case of immunotherapy alone, it would be necessary to avoid a long treatment that could increase the risk of disease progression. In this latter case, as a rule of thumb, I would limit the treatment to 1 month/1.5 months.

Antonio Rossi: Based on the available data and the design of the ongoing randomized trials, $2-4$ cycles of neoadjuvant single-agent immunotherapy or combined with platinumbased chemotherapy should be appropriate.

Dirk de Ruysscher: The best for the future may be ctDNA, but this needs more development and especially standardization. Advanced quantitative imaging methods based on e.g., deep learning algorithms show great promise.

Robert A. Ramirez: I agree that 2 cycles of neoadjuvant treatment be followed by imaging and a multidisciplinary review to see when the right time for surgery should be. If responding following 2 cycles but still borderline resectable, then an additional 2 cycles could be beneficial.

Wolfram C. M. Dempke: It depends on the early responses seen-if there is a response, we normally go for 4 cycles with a re-evaluation after two cycles.

D. Ross Camidge: To date 2-4 cycles are given but the stress of delaying surgery has to be weighed against data.

Nicolas Guibert: The number of cycles will depend on the approvals obtained in France. It will follow the regimen studied in the RCT that will lead to this approval. The most appealing data have however been observed with ICI and chemo combinations, with 3 to 4 cycles.

Raffaele Califano: We will consider a cycle 3 or 4 weeks of treatment.

\section{What adjuvant treatment regimen do/will you choose among those respond to neoadjuvant PD-1/PD-L1 blockade therapy?}

Rafael Rosell: For responding patients with complete or major pathological response, adjuvant immunotherapy for 1 year could be recommended.

Mariano Provencio: We use carbo-taxol-nivolumab.

Erminia Massarelli: I continue same immunotherapy plus or minus chemotherapy. However if MPR is significant I only continue immunotherapy if patients were treated with IO alone as induction.

Mara B. Antonoff: This depends on the stage of disease. For those with early-stage disease, no need for adjuvant therapy. However, for recurrent disease, node positive disease, or large tumors that would otherwise get adjuvant systemic therapy, immunotherapy should be given to those with elevated PD-L1 expression.

Toyoaki Hida: We choose ICI used in the preoperative treatment.

Marc de Perrot: PD-1/PD-L1 blockade will be maintained in the adjuvant setting.

Steven H. Lin: Although chemotherapy is not used adjuvant even if it is given concurrently with immunotherapy, only the immunotherapy as a single agent is used in the adjuvant setting for most trials. Radiotherapy is used per the discretion of the trial, with some global trials excluding the use of postoperative radiotherapy for IIIA-N2 disease resected to negative margins.

Massimo Di Maio: We have no data to produce evidencebased recommendations. For patients who have not received chemotherapy, I would consider adjuvant chemotherapy according to current guidelines. For patients who have received chemotherapy as neoadjuvant treatment (i.e., combo of chemo + immuno), probably they could receive no further systemic treatment after surgery. I prefer waiting for clinical trials. 
Antonio Rossi: Platinum-based doublets, if not received in neoadjuvant setting, should be considered the standardof-care with or without PD-1/PD-L1 inhibitors as adjuvant treatment. At the moment, this combination approach is reserved for patients enrolled in clinical trials.

Dirk de Ruysscher: In case of an MPR or a pCR: the same anti-PD-1/PD-L1 as pre-operatively. In case no MPR or pCR was achieved: (I) if only neoadjuvant immunotherapy: 4 cycles of a platinum doublet depending on the histology of the tumor; (II) if concurrent chemotherapy and immunotherapy: no further adjuvant therapy or no systemic therapy has shown a benefit.

Robert A. Ramirez: If the patient has not received neoadjuvant chemo and only received IO then if adjuvant therapy is indicated then I would use a cisplatin-based regimen based on histology.

Wolfram C. M. Dempke: We believe that the adjuvant treatment regimen should be the same as used for the neoadjuvant setting, however without chemotherapy if already used in the neoadjuvant part.

D. Ross Camidge: Trial specific at present.

Nicolas Guibert: Again, the adjuvant regimen will depend on the approvals obtained in France that will follow the main RCTs' outcomes. Most trials were however designed with ana approximately 1 -year adjuvant immunotherapy (13 additional pembrolizumab adjuvant cycles in the KEYNOTE 617,16 cycles of atezolizumab in the IMPOWER 030) and it is very likely that this approach may be the most suited. It will also certainly depend on the pathological response (CR, MPR) and definitive pathological stage (pTNM) after surgery.

Raffaele Califano: We will give platinum/vinorelbine $\times 4$ cycle.

\section{Acknowledgments}

The authors appreciate the academic support from AME Lung Cancer Collaborative Group.

Funding: None.

\section{Footnote}

Conflicts of Interest: All authors have completed the ICMJE uniform disclosure form (available at http://dx.doi. org/10.21037/tlcr-2020-63). The authors have no conflicts of interest to declare. The authors have no conflicts of interest to declare.
Ethical Statement: The authors are accountable for all aspects of the work in ensuring that questions related to the accuracy or integrity of any part of the work are appropriately investigated and resolved.

Open Access Statement: This is an Open Access article distributed in accordance with the Creative Commons Attribution-NonCommercial-NoDerivs 4.0 International License (CC BY-NC-ND 4.0), which permits the noncommercial replication and distribution of the article with the strict proviso that no changes or edits are made and the original work is properly cited (including links to both the formal publication through the relevant DOI and the license). See: https://creativecommons.org/licenses/by-nc-nd/4.0/.

\section{References}

1. Bray F, Ferlay J, Soerjomataram I, et al. Global cancer statistics 2018: GLOBOCAN estimates of incidence and mortality worldwide for 36 cancers in 185 countries. CA Cancer J Clin 2018;68:394-424.

2. Chen $W$, Zheng R, Baade PD, et al. Cancer statistics in China, 2015. CA Cancer J Clin 2016;66:115-32.

3. Molina JR, Yang P, Cassivi SD, et al. Non-small cell lung cancer: epidemiology, risk factors, treatment, and survivorship. Mayo Clin Proc 2008;83:584-94.

4. Arriagada R, Bergman B, Dunant A, et al. Cisplatinbased adjuvant chemotherapy in patients with completely resected non-small-cell lung cancer. $\mathrm{N}$ Engl J Med 2004;350:351-60.

5. Winton T, Livingston R, Johnson D, et al. Vinorelbine plus cisplatin vs. observation in resected non-small-cell lung cancer. N Engl J Med 2005;352:2589-97.

6. Douillard JY, Rosell R, De Lena M, et al. Adjuvant vinorelbine plus cisplatin versus observation in patients with completely resected stage IB-IIIA non-small-cell lung cancer (Adjuvant Navelbine International Trialist Association [ANITA]): a randomised controlled trial. Lancet Oncol 2006;7:719-27.

7. Goldstraw P, Chansky K, Crowley J, et al. The IASLC Lung Cancer Staging Project: Proposals for Revision of the TNM Stage Groupings in the Forthcoming (Eighth) Edition of the TNM Classification for Lung Cancer. J Thorac Oncol 2016;11:39-51.

8. Uprety D, Mandrekar SJ, Wigle D, et al. Neoadjuvant Immunotherapy for NSCLC: Current Concepts and Future Approaches. J Thorac Oncol 2020;15:1281-97. 
9. NSCLC Meta-analysis Collaborative Group. Preoperative chemotherapy for non-small-cell lung cancer: a systematic review and meta-analysis of individual participant data. Lancet 2014;383:1561-71.

10. Lim E, Harris G, Patel A, et al. Preoperative versus postoperative chemotherapy in patients with resectable non-small cell lung cancer: systematic review and indirect comparison meta-analysis of randomized trials. J Thorac Oncol 2009;4:1380-8.

11. Gandhi L, Rodriguez-Abreu D, Gadgeel S, et al. Pembrolizumab plus Chemotherapy in Metastatic NonSmall-Cell Lung Cancer. N Engl J Med 2018;378:2078-92.

12. Paz-Ares L, Luft A, Vicente D, et al. Pembrolizumab plus Chemotherapy for Squamous Non-Small-Cell Lung Cancer. N Engl J Med 2018;379:2040-51.

13. Mok TSK, Wu YL, Kudaba I, et al. Pembrolizumab versus chemotherapy for previously untreated, PD-L1expressing, locally advanced or metastatic non-small-cell lung cancer (KEYNOTE-042): a randomised, open-label, controlled, phase 3 trial. Lancet 2019;393:1819-30.

14. Hanna NH, Schneider BJ, Temin S, et al. Therapy for Stage IV Non-Small-Cell Lung Cancer Without Driver Alterations: ASCO and $\mathrm{OH}$ (CCO) Joint Guideline Update. J Clin Oncol 2020;38:1608-32.

15. Santarpia M, Aguilar A, Chaib I, et al. Non-Small-Cell Lung Cancer Signaling Pathways, Metabolism, and PD-1/ PD-L1 Antibodies. Cancers (Basel) 2020;12:1475.

16. Garon EB, Hellmann MD, Rizvi NA, et al. Five-Year Overall Survival for Patients With Advanced Non-SmallCell Lung Cancer Treated With Pembrolizumab: Results From the Phase I KEYNOTE-001 Study. J Clin Oncol 2019;37:2518-27.

17. Topalian SL, Hodi FS, Brahmer JR, et al. Five-Year Survival and Correlates Among Patients With Advanced Melanoma, Renal Cell Carcinoma, or Non-Small Cell Lung Cancer Treated With Nivolumab. JAMA Oncol 2019;5:1411-20.

18. O'Donnell JS, Hoefsmit EP, Smyth MJ, et al. The Promise of Neoadjuvant Immunotherapy and Surgery for Cancer Treatment. Clin Cancer Res 2019;25:5743-51.

19. Roth JA, Fossella F, Komaki R, et al. A randomized trial comparing perioperative chemotherapy and surgery with surgery alone in resectable stage IIIA non-small-cell lung cancer. J Natl Cancer Inst 1994;86:673-80.

20. Rosell R, Gomez-Codina J, Camps C, et al. A randomized trial comparing preoperative chemotherapy plus surgery with surgery alone in patients with non-small-cell lung cancer. N Engl J Med 1994;330:153-8.

21. Song WA, Zhou NK, Wang W, et al. Survival benefit of neoadjuvant chemotherapy in non-small cell lung cancer: an updated meta-analysis of 13 randomized control trials. J Thorac Oncol 2010;5:510-6.

22. Keir ME, Butte MJ, Freeman GJ, et al. PD-1 and its ligands in tolerance and immunity. Annu Rev Immunol 2008;26:677-704.

23. Liu J, Blake SJ, Yong MC, et al. Improved Efficacy of Neoadjuvant Compared to Adjuvant Immunotherapy to Eradicate Metastatic Disease. Cancer Discov 2016;6:1382-99.

24. Bourgeois-Daigneault MC, Roy DG, Aitken AS, et al. Neoadjuvant oncolytic virotherapy before surgery sensitizes triple-negative breast cancer to immune checkpoint therapy. Sci Transl Med 2018;10:eaao1641.

25. Brooks J, Fleischmann-Mundt B, Woller N, et al. Perioperative, Spatiotemporally Coordinated Activation of $\mathrm{T}$ and NK Cells Prevents Recurrence of Pancreatic Cancer. Cancer Res 2018;78:475-88.

26. Forde PM, Chaft JE, Smith KN, et al. Neoadjuvant PD-1 Blockade in Resectable Lung Cancer. N Engl J Med 2018;378:1976-86.

27. Kwiatkowski DJ RV, Chaft JE, BE J. Neoadjuvant atezolizumab in resectable nonsmall cell lung cancer (NSCLC): Interim analysis and biomarker data from a multicenter study (LCMC3). J Clin Oncol 2019;37:abstr 8503.

28. Ready N, Tong B, Clarke J, et al. Neoadjuvant Pembrolizumab in Early Stage Non-Small Cell Lung Cancer (NSCLCL): Toxicity, Efficacy, and Surgical Outcomes. J Thorac Oncol 2019;14:S745.

29. Wislez M, Mazieres J, Lavole A, et al. Neoadjuvant durvalumab in resectable non-small cell lung cancer (NSCLC): preliminary results from a multicenter study (IFCT-1601 IONESCO). Ann Oncol 2020;31:S794.

30. Gao S, Li N, Gao S, et al. Neoadjuvant PD-1 inhibitor (Sintilimab) in NSCLC. J Thorac Oncol 2020;15:816-26.

31. Besse B, Adam J, Cozic N, et al. Neoadjuvant atezolizumab (A) for resectable non-small cell lung cancer (NSCLC): results from the phase II PRINCEPS trial. Ann Oncol 2020;31:S735-43.

32. Shu CA, Gainor JF, Awad MM, et al. Neoadjuvant atezolizumab and chemotherapy in patients with resectable non-small-cell lung cancer: an open-label, multicentre, single-arm, phase 2 trial. Lancet Oncol 2020;21:786-95.

33. Provencio $M$, Nadal E, Insa A, et al. Neoadjuvant chemotherapy and nivolumab in resectable non-small-cell 
lung cancer (NADIM): an open-label, multicentre, singlearm, phase 2 trial. Lancet Oncol 2020;21:1413-22.

34. Xia L, Liu Y, Wang Y. PD-1/PD-L1 Blockade Therapy in Advanced Non-Small-Cell Lung Cancer: Current Status and Future Directions. Oncologist 2019;24:S31-S41.

35. Reck M, Rodriguez-Abreu D, Robinson AG, et al. Pembrolizumab versus Chemotherapy for PD-L1Positive Non-Small-Cell Lung Cancer. N Engl J Med 2016;375:1823-33.

36. Spigel D, de Marinis F, Giaccone G, et al. IMpower110: Interim overall survival (OS) analysis of a phase III study of atezolizumab (atezo) vs platinum-based chemotherapy (chemo) as first-line (1L) treatment (tx) in PD-L1-selected NSCLC. Ann Oncol 2019;30:v851-v934.

37. Available online: https://wwwfdagov/news-events/pressannouncements/fda-approves-first-cancer-treatment-anysolid-tumor-specific-genetic-feature

38. Hellmann MD, Ciuleanu TE, Pluzanski A, et al. Nivolumab plus Ipilimumab in Lung Cancer with a High Tumor Mutational Burden. N Engl J Med 2018;378:2093-104.

39. Available online: https://wwwfdagov/drugs/drugapprovals-and-databases/fda-approves-pembrolizumabadults-and-children-tmb-h-solid-tumors

40. Cappuzzo F, Reck M, Socinski MA, et al. IMpower150: Exploratory analysis of brain metastases development. J Clin Oncol 2020;38:abstr 9587.

41. Zhou J, Tang Z, Gao S, et al. Tumor-Associated Macrophages: Recent Insights and Therapies. Front Oncol 2020;10:188.

42. Ford K, Hanley CJ, Mellone M, et al. NOX4 Inhibition Potentiates Immunotherapy by Overcoming CancerAssociated Fibroblast-Mediated CD8 T-cell Exclusion from Tumors. Cancer Res 2020;80:1846-60.

43. Tina Cascone, William Nassib William, Annikka Weissferdt, et al. Neoadjuvant nivolumab (N) or nivolumab plus ipilimumab (NI) for resectable non-small cell lung cancer (NSCLC): Clinical and correlative results from the NEOSTAR study. J Clin Oncol 2019;37:abstr 8504.

44. Hanahan D, Weinberg RA. Hallmarks of cancer: the next generation. Cell 2011;144:646-74.

45. Borghaei H, Paz-Ares L, Horn L, et al. Nivolumab versus Docetaxel in Advanced Nonsquamous Non-Small-Cell Lung Cancer. N Engl J Med 2015;373:1627-39.

46. Cascone T, William WN, Weissferdt A, et al. Pembrolizumab versus docetaxel for previously treated, PD-L1-positive, advanced non-small-cell lung cancer
(KEYNOTE-010): a randomised controlled trial. Lancet 2016;387:1540-50.

47. Bylicki O, Paleiron N, Margery J, et al. Targeting the PD-1/PD-L1 Immune Checkpoint in EGFR-Mutated or ALK-Translocated Non-Small-Cell Lung Cancer. Target Oncol 2017;12:563-9.

48. Skoulidis F, Arbour KC, Hellmann MD, et al. Association of STK11/LKB1 genomic alterations with lack of benefit from the addition of pembrolizumab to platinum doublet chemotherapy in non-squamous non-small cell lung cancer. J Clin Oncol 2019;37:abstr 102.

49. Mutations Associated with Sensitivity or Resistance to Immunotherapy in mNSCLC. Analysis from the MYSTIC Trial. Available online: https://www.eurekalert.org/pub_ releases/2019-09/iaft-maw090819.php

50. Cho BC, Lopes G, Kowalski DM, et al. Relationship between STK11 and KEAP1 mutational status and efficacy in KEYNOTE-042: pembrolizumab monotherapy versus platinum-based chemotherapy as first-line therapy for PDL1-positive advanced NSCLC. AACR 2020;80:abstr CT084.

51. Nadal E, Heeke S, Benzaquen J, et al. Two Patients With Advanced-Stage Lung Adenocarcinoma With Radiologic Complete Response to Nivolumab Treatment Harboring an STK11/LKB1 Mutation. JCO Precis Oncol 2020;4:1239-45.

52. Uprety D, Mandrekar SJ, Wigle D, et al. Neoadjuvant Immunotherapy for NSCLC: Current Concepts and Future Approaches. J Thorac Oncol 2020;15:1281-97.

53. Liu J, O’Donnell JS, Yan J, et al. Timing of neoadjuvant immunotherapy in relation to surgery is crucial for outcome. Oncoimmunology 2019;8:e1581530.

54. Amaria RN, Menzies AM, Burton EM, et al. Neoadjuvant systemic therapy in melanoma: recommendations of the International Neoadjuvant Melanoma Consortium. Lancet Oncol 2019;20:e378-89.

55. Travis WD, Dacic S, Wistuba I, et al. IASLC Multidisciplinary Recommendations for Pathologic Assessment of Lung Cancer Resection Specimens After Neoadjuvant Therapy. J Thorac Oncol 2020;15:709-40.

56. William WN Jr, Pataer A, Kalhor N, et al. Computed tomography RECIST assessment of histopathologic response and prediction of survival in patients with resectable non-small-cell lung cancer after neoadjuvant chemotherapy. J Thorac Oncol 2013;8:222-8.

57. Tao X, Li N, Wu N, et al. The efficiency of (18)F-FDG PET-CT for predicting the major pathologic response to the neoadjuvant PD-1 blockade in resectable non- 
small cell lung cancer. Eur J Nucl Med Mol Imaging 2020;47:1209-19.

58. Huang C, Liu S, Tong X, et al. Extracellular vesicles and ctDNA in lung cancer: biomarker sources and therapeutic applications. Cancer Chemother Pharmacol 2018;82:171-83.

59. Chae YK, Oh MS. Detection of Minimal Residual Disease Using ctDNA in Lung Cancer: Current Evidence and Future Directions. J Thorac Oncol 2019;14:16-24.

60. Akondy RS, Fitch M, Edupuganti S, et al. Origin and differentiation of human memory CD8 T cells after vaccination. Nature 2017;552:362-7.

61. Edelman MJ, Suntharalingam M, Burrows W, et al. Phase I/II trial of hyperfractionated radiation and chemotherapy followed by surgery in stage III lung cancer. Ann Thorac Surg 2008;86:903-10.

62. Shah AA, Berry MF, Tzao C, et al. Induction chemoradiation is not superior to induction chemotherapy alone in stage IIIA lung cancer. Ann Thorac Surg 2012;93:1807-12.

63. Bott MJ, Cools-Lartigue J, Tan KS, et al. Safety and Feasibility of Lung Resection After Immunotherapy for Metastatic or Unresectable Tumors. Ann Thorac Surg 2018;106:178-83.

64. Junker K, Thomas M, Schulmann K, et al. Tumour regression in non-small-cell lung cancer following neoadjuvant therapy. Histological assessment. J Cancer Res Clin Oncol 1997;123:469-77.

65. Junker K, Langner K, Klinke F, et al. Grading of tumor regression in non-small cell lung cancer : morphology and prognosis. Chest 2001;120:1584-91.

Cite this article as: Liang W, Cai K, Chen C, Chen H, Chen Q, Fu J, Hu J, Jiang T, Jiao W, Li S, Liu C, Liu D, Liu W, Liu Y, Ma H, Pan X, Qiao G, Tian H, Wei L, Zhang Y, Zhao S, Zhao X, Zhou C, Zhu Y, Zhong R, Li F, Rosell R, Provencio M, Massarelli E, Antonoff MB, Hida T, de Perrot M, Lin SH, Di Maio M, Rossi A, De Ruysscher D, Ramirez RA, Dempke WCM, Camidge DR, Guibert N, Califano R, Wang Q, Ren S, Zhou C, He J. Expert consensus on neoadjuvant immunotherapy for non-small cell lung cancer. Transl Lung Cancer Res 2020;9(6):2696-2715. 10.21037/tlcr-2020-63
66. Qu Y, Emoto K, Eguchi T, et al. Pathologic Assessment After Neoadjuvant Chemotherapy for NSCLC: Importance and Implications of Distinguishing Adenocarcinoma From Squamous Cell Carcinoma. J Thorac Oncol 2019;14:482-93.

67. Owen D, Chaft JE. Immunotherapy in surgically resectable non-small cell lung cancer. J Thorac Dis 2018;10:S404-S11.

68. Antonia SJ, Villegas A, Daniel D, et al. Durvalumab after Chemoradiotherapy in Stage III Non-Small-Cell Lung Cancer. N Engl J Med 2017;377:1919-29.

69. Eberhardt WE, Pottgen C, Gauler TC, et al. Phase III Study of Surgery Versus Definitive Concurrent Chemoradiotherapy Boost in Patients With Resectable Stage IIIA(N2) and Selected IIIB Non-Small-Cell Lung Cancer After Induction Chemotherapy and Concurrent Chemoradiotherapy (ESPATUE). J Clin Oncol 2015;33:4194-201.

70. Reck M, Ciuleanu T, Cobo M, et al. First-line nivolumab (NIVO) + ipilimumab (IPI) combined with 2 cycles of platinum-based chemotherapy (chemo) vs 4 cycles of chemo in advanced non-small cell lung cancer (NSCLC): Patient-reported outcomes (PROs) from CheckMate 9LA. Ann Oncol 2020;31:S1142-215.

71. Provencio M, Nadal E, Insa A, et al. NEO-adjuvant chemo-immunotherapy for the treatment of STAGE IIIA resectable non-small-cell lung cancer (NSCLC): A phase II multicenter exploratory study-Final data of patients who underwent surgical assessment. J Clin Oncol 2019;37:abstr 8509. 
Table S1 The ongoing trials of neadjivant immunotherapy

\begin{tabular}{ll}
\hline Study title & NCT.no \\
\hline A Singl-arm, phase IIIttudy yf Neoadivant MPDL3280A, Nab-pacilitaxel and Carboplatin (MAC) in NCT02716038 \\
Resectable Non-small Cell Lung Cancer (NSCLC)
\end{tabular}

Randomized, Open-label, Controlled Phase III Trial Comparing Pembrolizumab-platinum Based
Chemotherapy Combination With Pembrolizumab Monstion Chemotherapy Combination With Pembrolizumab Monotherapy
small-cell Lung Cancers (NSCLC) With PDLL Expression $250 \%$

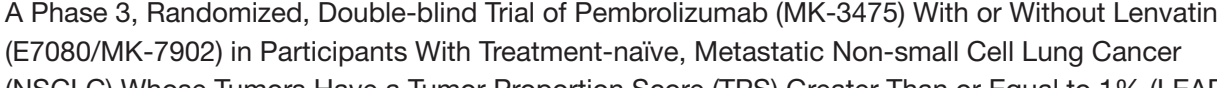
(NSCLC) Whose Tumors Hays a 10
007)-China Extension Study

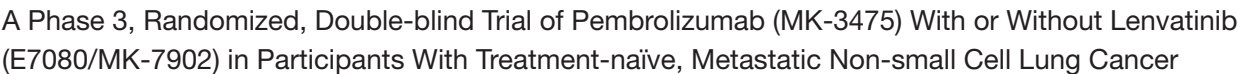

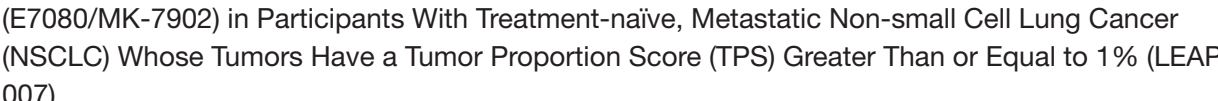

A Randomized, Phase 3 Trial With Anti-PD-1 Monoclonal Antibody Pembrolizumab (MK-3475)
Versus Placebo for Patitints With Early Stage NSCLC After Resection and Completion of Standard A Phase 3, Randomized, Placebo-Controlled Clinical Study to Evaluate the Safety and Efficacy of Stereotactic Body Radiotherapy (SBRT) With or Without Pembrolizumab (MK-3475) in Participants
With Medically Inoperable Stages I or III Non Small Cell Lung Cancer (NSCLC) (KEYNOTE-867) A Phase 3, Multicenter, Randomized, Open-label Irial to Compare the Efficacy and Sarety of
Pembrolizumab (MK-3475) in Combination With Lenvatinib (E7080/MK-7902) Versus Docetaxel in Previously Treated Participants With Metastatic Non-small Cell Lung Cancer (NSCLC) and A Randomized, Double-Blind, Phase 3 Study of Pemetrexed + Platinum Chemotherapy With or

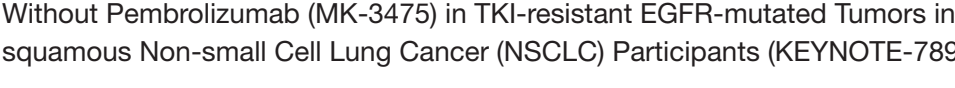

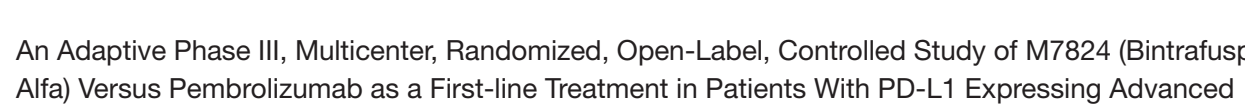
Affa) Versus Pembroilizumab as a First-line Treatment in Patients With PD-LL Expressing Advanced
Non-small Cell Lung Cancer A Phase III, Randomized, Double-blind Trial of Platinum Doublet Chemotherapy + -Pembrolizumaa
(MK-3475) as Neoadijuant/Adjuant Therapy for Participants With Resectable Stage II, IIIA, and A Randomized, Double-blind, Placebo-controlled, Phase III Study Evaluating the Efficacy and Safety
of Pembrolizumab Plus Platinum-based Doublet Chemotherapy With or Without Canakinumab as First Line Therapy for Locally Advanced or Metastatic Non-squamous and Squamous Non-smal

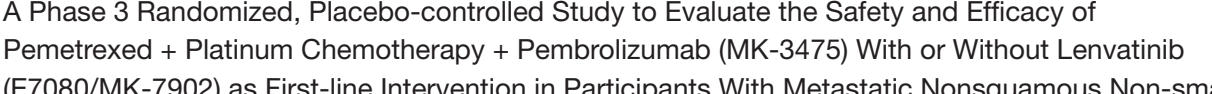
(E7080/MK-7902) as First-lin
Cell Lung Cancer (LEAP-006

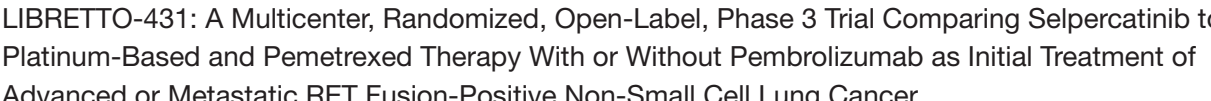
A Randomized, Double-Blind, Phase III Study of Carboplatin-Pacitizel/Nab-Paclitaxe small Cell Lung Cancer Subjects (KEYNOTE-407) A Phase 3 Study of Pembrolizumab in Combination With Carboplatin/Taxane (Pacilizexl or Nab-
pacilitaxel) Followed by Pembrolizumab With or Without Manitenance Olaparibi in the First-line

PD-1 1 Inibititors and Chemothe
Non-small Cell Lung Cancer

A Phase 3 Study of Pembrolizumab (MK-3475) in Combination With Concurrent Chemoradiation

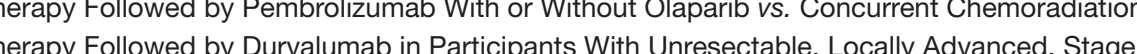
Non-Small Cell Lung Cancer (NSCLC)

A Phase 3 Study of Pembrolizumab in Combination With Pemetrexed/Platinum (Carboplatin or Cisplatin) Followed by Pembrolizumab and Maintenance Olaparib vs. Maintenance Pemetrexed in
the First-Line Treatment of Participants With Metastatic Nonsquamous Non-Small-Cell Lung Cancer

A Randomized Trial of Consolidativiv Immunotherapy With vs. Without Thoracic Radiotherapy and
I Or Stereotactic Body Radiation Therapy (SBRT) After First-Line Systemic Therapy for Metastatic
NSCLC

A Randomized, Phase 3, Open-Label Study of Combinations of REGAN2810 (Anti-PD-1 Antibody),

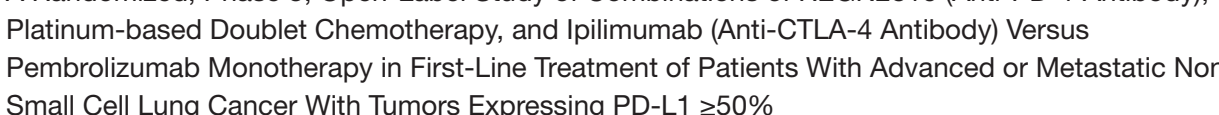

QULLT 2.023: A Phase 3, Dpen-Label, 3-Cohort Randomized Study of N-803, in Combination With
Current or Metastatic NSCLC.

A Randomized, Open-Label, Phase 3 Studd of Pralsetinib Versus Standard of Care for First Line
Treatment of RET Fusion-postitive, Metastataic Non-Small Cell Lung Cancer

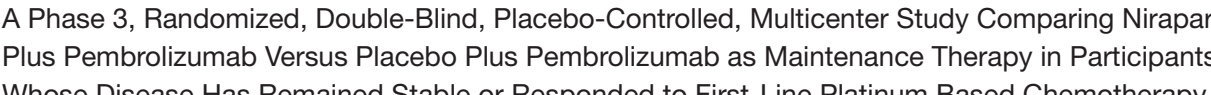
Whose Disease Has Remained Stable or Responded to First-line Platitum Based Chemotherapy
With Pembrorizumab for Stage IIIB or IV Non-Small Cell Lung Cancer A Randomized Non-inferiority Trial Evaluating the Length of Treatment With PD-1/PD-LL I nhibitiors NCT0415798 An Open-label, Randomized Phase III Study of Early Switch Maintenance vs. DElayed Second-
line Nivolumab in Advanced Stage Squamous Non Small-cell Lung Cancer (NSCLC) Patients After Standard First-line Platinum-based Chemotherapy - EDEN Trial A Phase 3, Randomized, Open Label Study to Compare Nivolumab Plus Concurrent
Chemoradiotherapy (CCRT) Followed by Nivolumab Plus plilimumab or Nivolumab Plus CCRT Chemoradiotherapy (CCRT) Followed by Nivolumab Plus Ipilimumab or Nivolumab Plus CCFT
Followed by Nivolumab s. CCRT Followed by Durvalumab in Previously Untreated, Locally
Advanced Non-small Cell Lung Cancer (LA NSSCC) A Phase IIIL/IV Safety Trial of Nivolumab (BMS-936558) in Subjects With Advanced or Metastatic
Non-Small

An Open-Label, Randomized Phase 3 Trial of Nivolumab, or Nivolumab Plus Ipilimumab, or Nivolumab Plus Platinum Doublet Chemotherapy Versus Platinum Doublet Chemetherapy in
Subjects With Chemotherapy-Naive Stage lV or Recurrent Non-Small Cell Lung Cancer (NSCLO

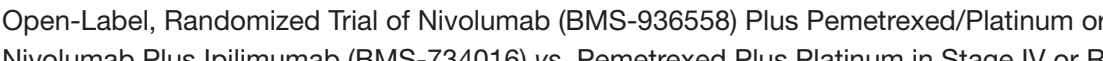

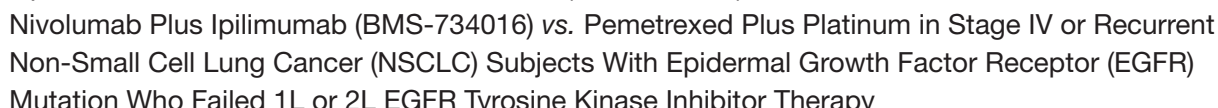
Randomized, OpenLLabel, Phase 3 Trial of Nivolumab Plus Ipilimumab or Nivolumab Plus
Doublet Chemotherapy Versus Platinum Doublet Chemotherapy in Early Stage NSCLC

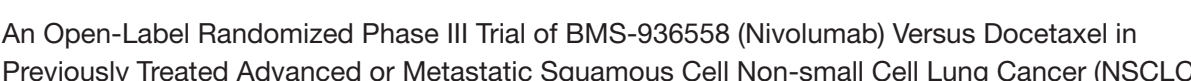
Randomized Phase III Study Testing Nivolumab and Ipilimumab Versus a Carboplatin Based
Doublet in First Line Treatment of PS 2 or Elderly (More Than 70 Years Old) Patients With Advan

An Open Label, Sarety Study yof Participants With Non-Small Cell Lung Cancer Receiving Second-
Line Nivolumab Monotherapy in Asia

A Randomized Phase 3 Study of Sitravatinibi in Combination With Nivolumab Versus Docetaxel in
Patients With Advanced Non-Squarous Non-Small Cell Lung Cancer With Disease Progression

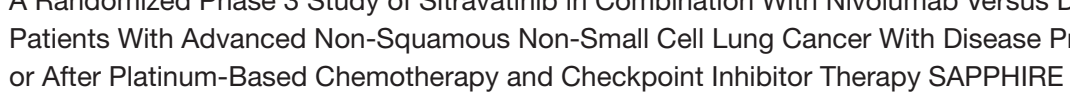

A Randomized Phase 3 Trial Comparing Continuation Nivolumab-lilimumab Doublet
Immunotherapy Until Progression Versus Observation in Treatment-naive Patients With PDLImmunotherapy Untili Progression Versus Observation in Treatment-naive Patients With PDL1
positive Stage IV Non-Small Cell Lung Cancer (NSCLC) After Nivolumab-piliinumabab Induction

Adjuvant Nivolumab in Resected Lung Cancers (ANVIL) - A Randomized Phase III Study of
Nivolumab After Surgical Resection and Adjuvant Chemotherapy in Non-Small Cell Lung Cancers Randomized Phase III Tiala of Local Consolidation Therapy (LCT) Atter Nivolumab and Ipilimuma
for IImmunotherapy-avive Patients With Metastatic Non-Small Cell Lung Cancer (LONESTAR)
-Strategic Alliance: BMS

A Phase III Clinical Trial of Adjuvant Chemotherapy vs. Chemoimmur
Completely Resected Non-small Cell Lung Cancer (NSCLC) Patients.

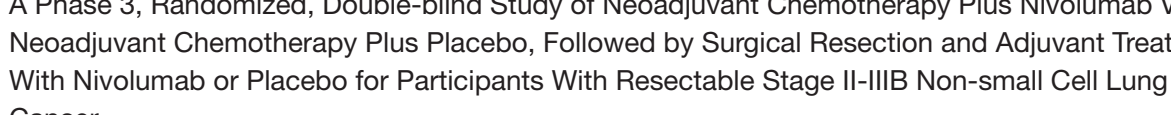
A Multicenter, Randomized, Double-Blind Trial in Subjects With Non-Squamous Non-Small Cell
Lung Cancer (TASUKK-52)

NCT04547504

NCT03924869

NCт0397637

NCT03515837

NCT0438063

NCT03976323

NCT0386717

NCT03515629

NCT03520686

NCT04222972

CT040264

NCT0286425
Key inclusion and exclusion criteria Drug

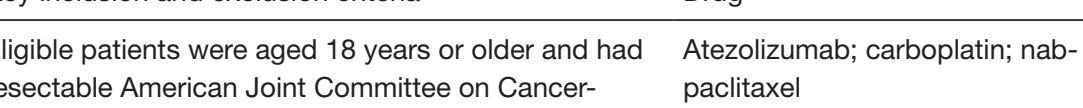
defined Stage 1 B-IIIA non--small-cell llung cancer, an
ECOG performance status of $0-1$, and a history of 作 Advanced NSCLC molecularly def
expression $250 \%$ of of tumour cells

No prior systemic theanement

\section{Pembrolizumab; isplatin
carboplatin AUC}

whose tumors has a PD-L1 TPS $21 \%$

No prior systemic therapy for their me
whose tumors has a PD-L1 TPS $\geq 1 \%$

NCT02504372 $\begin{array}{ll}\text { Staas } \\ \text { rese }\end{array}$

platinum doublet chemotherapy and treatment with
one prior anti-PD-1/PD-L1

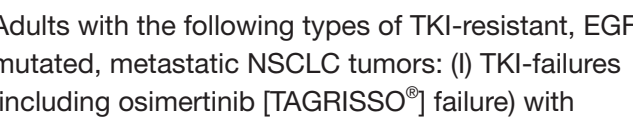

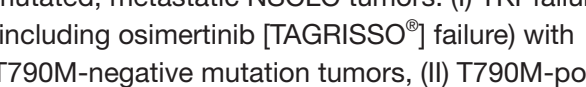

mutation tumors with prior exposure to osimertiniti
and (illif first-line osimertinib failure regarcless of

\section{Thom mutation status}

Advanced NSCLC with high PD-L1-tumor exp
with no EGFR mutation or ALK translocation

Resecta
NSCLC

Previously untreated locally advanced or metastatic
non-squamous and squamous NSCLC Subjects

Ther

NCT03829319 Adults with metastatic nonsquamous NSCLC

Participants with RET fusion-positive non-squamous
NSSLC that has spread to other parts of the body

Chinese adults with first line metastatic squamous
NSCLC

Have not received prior systemic treatment for their
advanced/metastatic NSCLC

Patients with advanced (stage IIIB/IY) NSCLC

NSCLC

Patients with advanced squamous or non-squamous
NSCLC whose tumors express PD-LL in $250 \%$ of

tumor cells

Patients with RET fusion-positive metastatic NSCLC Who have not previously recectur Participants with Stage IIIB or IV NSCLC who have achieved Stable disease (SD), Partial response (PP)
or completer response (CR) following completions or complete response (CR) following completion of
platinum-based first-ine induction chemotherapy with Patients who have disease stability to stop treatment
at 1 year or continue treatment until disease

Patients with advanced stage squamous NSCLC
without immunotherapy

$\begin{array}{ll}\text { NCTO2066636 } & \begin{array}{l}\text { Advanced or metastatic NSCLC (subjects with } \\ \text { non-squamous histology must be tested for EGFR }\end{array} \\ \text { nat }\end{array}$ non-squamous histology must be
mutations and ALK rearrangemenen

NCT02477826 Stage IV or recurrent NSCLC, with no prior systemic

Patients with EGFR mutation, NSCLC who failed firs
Ine (1L) or second-line (2L) EGFR TKL therapy Early stage IB-IIIA, operable NSCLC

Subjects with squamous cell NSCLC, after failure of prior platinum-based chemotherapy

Patients already have metastatic disease and a systemic, palliative treatment is the primary therapen
option Patients in Asia with NSCLC who are treated with
Nivolumab monotherapy as a second line or third line treatment Patients with advanced non-squamous NSCLC who
have previously experienced disease progression on or after platinumumbased chemotherapy and checkpoint
inhibitor therapy

Stage IV NSCLC, with no prior systemic anticancer
therapy, PD-LL tumor content $\geq 1 \%$ and $<50 \%$

Canakinumab; pembrolizumab carboplatin; ;isplatin; paclitaxid
nab-paclitaxel; pemetrexed

Pembrolizumab; carboplatin;
cisplatin; pemetrexed; lenvatinib

Selpercatinib; carboplatin; cisplatiti
pemetrexed; pembrolizumab

Pembrolizumab; paclitiaxel; nab-
pacilitaxel; carbopplatin

Pembrolizumab; carboplatin;
paclitaxel; nab-paclitaxel; olapa

Radiotherapy; pembrolizumab;
chemotherapy

Pembrolizumab + chemotherany

Pembrolizumab; olaparib;

etoposide; carboplatin; cisplatitis
pacilitael; pemetrexed; thoracic
radiotherapy; durvalumab

embrolizumab; pemetrexed

Stereotactic bod

REGN2810/ipi; REGN281//chemo/ Pembrolizuma

N-803; pembroliz
nab-paclitaxel

n; Control A: pembrolizumab; Control
B: carboplatin + nab-pacilitaxel
or paclitiaxel + pembrolizumab. control c: cisplatin or carboplatr

Pralsetinib; carboplatin; cisplatin;
pemetrexed; pembrolizumab;

gemitiabine

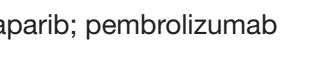

embrolizuma

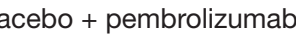

$\begin{array}{ll}\text { Pembrolizumab; nivolumab; } & \text { Con } \\ \text { atezolizumab; iplimumab } & \text { inh }\end{array}$

Continue Treatment with PD-1/PD-11
hibitior

Best supportive care

Nivolumab $\quad$ Best supportive care

Nivolumab/durvalumab + CCRT

Nivolumab

Nivolumab; iplilimumab; carboplatin; Platinum doublet chemotherapy cisplatin; $g$
paclitaxel

Nivolumab; iplilimumab;

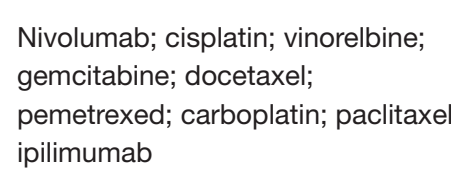

Nivolumab; docetaxe

Docetax Nivolumab; ipilimumab; $\quad$ Chemotherapy

\section{Nivolumab}

Ipilimumab; nivolumab

\section{Standard treatment}

months of treatment by nivo
tipilimumab then nivolumab ipilimumab then in case of
pogression platinum-based doub

NCT02595944 Patients with stage IB-IIIA NSCLC

Nivolumab

No intervention

Radiation therapy; local
consolidation therapy; nivolumab;
pilimumab

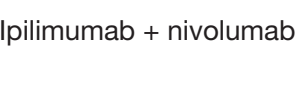

NCT04564157 Stage IB-IIIA, completely resected, NSCLC patients Carboplatin; paclitaxel; nivolumab Carboplatin + paclitaxe

Nivolumab; carboplatin; cisplatin;
paclitaxel; pemenerexed; doceataxel
chemo followed by Adj.Plac.

$\begin{array}{lll}\text { NCTO3117049 } & \begin{array}{l}\text { Stage IIII/N or recurrent non-squamous NSCLC } \\ \text { unsuitable for radical radiation }\end{array} & \begin{array}{l}\text { ONO-4538; carboplatin; pacilitaxel; } \\ \text { bevacizumab }\end{array}\end{array}$
Primary and secondary endpoint

The primary endpoint was major pathological

response, defined as the presence of $10 \%$ or
residual viable tumour at the time of surgen

The primary endpoint was PFS. The secondary
endpoints were PFS, PFS, ORR, OS, DOT, DOF,
AE and AESI

The primary endpoints were PFS and OS. The
secondary endpoints were ORR, AE, GHS, QoL,

The primary endpoints were PFS and OS. The
secondary endpoints were ORR, AE, GHS, QoL,

The primary endpoint was DFS
endpoints were OS and LCSS

The primary endpoints were EFS and OS. The secondary endpoints were TDDM, AE
EORTC QLQ-LC13/-C30 SCORE

The primary endpoints were OS and PFS. The

secondary endpoints were $\mathrm{ORR}, \mathrm{DOR}, \mathrm{AE}$, EORTC QLQ-LC13/C30 SCORE and TDD in

The primary endpoints were OS and PFS. The

secondary endpoints were ORR, DDR, AE,
EORTC QLQ-LC13/C30 SCORE and MDD in the

EORTC Questionnaire Composite Endpoint of

The primary endpoints were OS and PFS. The
secondary endpoint were TEAES, NCI-CTCAE, secondary endpoint were TEAEs, NCI-CTCAE
CR, PR and Immunogenicity

The primary endpoints were EFS and OS. The The primary endpoints were EFS and OS. The
secondary endpoints were mPR rate, $\mathrm{pCR}$ rate,
AE and EGHS/QLL SCORE

The primary endpoint were DLTs, PFS and OS.
The secondary endpoints were ORR, DCR, DO The seco
and TTR

The primary endopints were DLTS, AES, PFS and
OS. The secondary endpoints were ORR, DOR

The primary endpoint was PFS. The secondary
endionints were ORR, DCR, PFS, OS, DOR and The primary endpoints were PFS and OS. The

The primary endpoints were PFS and OS. The
secondary endpoints were AE, EORTC QLQ-C3 The primary endpoint was OS. The secondary
endpoints were Tumour ressonse, PFS, Acute/ Late toxicitites and Quality of life of the patients
using EORTC-QLQ-C30 The primary endpoints were PFS and OS. The
secondary yndpoitsw were AE, DOR, ORR and
EORTC-QLQ-C30 score

The primary endpoints were PFS and OS. The
secondary endpoints were AE, EORTC-QLQ-C secondary endpoints were AE, EORTC-OLQ-CB3
score and TID in EORTC QLQ-LC13 Cough (Item
311) Scale Score

The primary endpoint was PFS. The secondary
endpoints were Time of Progression, AE, Rate of Failure and Number of Particicipants with New
Sites of Disease The primary endpoint was PFS. The secondary
endpoints were OS, ORR, TEAES, SAES, DLTS
and Quality of fife

The primary endpoint was PFS. The secondary
endipoints were OS, ORR, PFS, DCR and Quality
of life

The primary endpoint was PFS. The secondary
endpoints were OS, ORR, AE, DCR and CBR The primary endpoints were PFS and OS. The
secondary yndpoitts were AE, TTD in Lung
Symptoms and TPP

The primary endpoint was Time to next treatment The secondary endpoints were OS, irAEs and
BOR

The primary endpoint was OS. The secondary
endpoints were PFS, TFF, OSind and PFSind The primary endpoints were PFS and OS. The
secondary endpoints were AE, ORR and TTR

The primary endpoint was AEs. The secondary
endpoint were AEs and Median time to onset and The primary endpoints were PFS and OS. The

The primary endpoint was PFS. The secondary
endooints were PFS, ORR, OS and DOR

The primary endpoints were EFS and $\mathrm{PCR}$. The
secondary endpoints were OS, MPR and TTDM

The primary endpoint was OS. The secondary
endpoints were PFS, ORR, OS and TRR

The primary endpoint was OS. The secondary
enddoints were PFS, ORR, Quality of life score

The primary endpoint was AE. The secondary

endpoints were AE and Laboratory test

The primary endpoint was OS. The secondary
endpoints were AEs, PFS and ORR

The primary endpoint was PFS. The secondary
endooints were OS, PFS and Quality of life

The primary endpoints were DFS and OS. The The primary endpoint was OS. The secondary
endpoints were PFS, TANM and Quality of life

The primary endpoint was DFS. The secondary
endpoints were OS and AE

The primary endpoint was EFS. The secondary
endpoints were OS, DCR, MPR and AE

The primary endpoint was PFS. The secondary
endpoints were OS, PFS, ORR and AE 